\title{
AUDITORY AND VISUAL MAPS OF SPACE IN THE OPTIC TECTUM OF THE OWL ${ }^{1}$
}

\author{
ERIC I. KNUDSEN
}

Department of Neurobiology, Stanford University School of Medicine, Stanford, California 94.305

Received January 25, 1982; Revised April 15, 1982; Accepted April 20, 1982

\begin{abstract}
The receptive field properties and functional organization of visual and auditory responses were studied in the optic tectum of the barn owl (Tyto alba). Most units throughout the depth of the tectum responded to both visual and auditory stimuli. The entire visual field of each eye was represented topographically in the contralateral tectum. In the portion of the tectal map representing the zone of binocular vision, $50 \%$ of the superficial layer units and $100 \%$ of the deep layer units were driven binocularly. The representation of the frontal binocular region of space was greatly expanded in the map; the average magnification factor was 3 times greater for the frontal binocular zone than for the monocular zone.

The responses of the superficial and deep tectal units to auditory stimuli were space specific; they responded only when a sound source was located in a particular region of space, or receptive field, regardless of the intensity or type of sound used. Most auditory receptive fields contained a distinct "best area" where a sound source was most effective in driving the unit. Auditory space, as defined by receptive fields and best areas, was represented topographically in the tectum.

The auditory and visual maps of space had the same orientations, positions, magnification factors, and termination coordinates at the anterior and dorsal edges of the tectum. Yet the maps lost their registry near the posterior and ventral margins where the most peripheral regions of space were represented. These characteristics suggest that the spatiotopic organization in the tectum is a compromise between a tendency for the space representations of different modalities to align and for the representation of each modality to fill the entire tectum.
\end{abstract}

The optic tectum, or superior colliculus, is a multimodal integration center in the vertebrate central nervous system that issues motor commands to orient the eyes, ears, and head of an animal toward stimuli of interest. Although the visual system serves as its primary source of sensory input, other "exteroceptive" systems also contribute, including the auditory and somatosensory systems in most vertebrates (Horn and Hill, 1966; Cynader and Berman, 1972; Gordon, 1973; Dräger and Hubel, 1975; Stein et al., 1976; Chalupa and Rhoades, 1977; Gaither and Stein, 1979), the infrared systems of pit vipers (Hartline et al., 1978), and the lateral line systems of fishes (Bastian, 1981). The extent and influence of these extravisual sources of input seem to vary

\footnotetext{
${ }^{1}$ I thank Phyllis Knudsen for help in every phase of this research. Also, I gratefully acknowledge the constructive comments and criticisms provided by Steven Esterly, Leslie Henderson, Masakazu Konishi, John Middlebrooks, Andrew Moiseff, Jane Norris, and Michael Stryker. This work was supported by grants from the March of Dimes Foundation (5-285), the National Institutes of Health (5 R01 NS 1609902), and W. M. Hume and by a Biomedical Research Support Grant (NIH 5S07 RR 5353-18).
}

with the importance of the modality in guiding the orientation behaviors of the particular species.

The sensory epithelium of each sensory system, with the notable exception of the auditory system, is represented in the tectum topographically so that the inputs from these various modalities appear as superimposed maps of the respective sensory epithelia. For example, the temporal portion of the contralateral retina projects in the tectum anteriorly (except in primate mammals; Cynader and Berman, 1972; Lane et al., 1973), the nasal portion posteriorly, the ventral portion medially, and the dorsal portion laterally. This gives rise to the systematic representation of contralateral visual space that has been documented exhaustively in many species. Similarly, the input of the somatosensory system originates from the contralateral body surface and also projects topographically to the tectum: facial regions project anteriorly, hind quarters posteriorly, axial regions medially, and distal extremities laterally (Dräger and Hubel, 1975; Stein et al., 1976). The same rule of topographic projection also holds true for infrared and lateral line inputs.

However, this rule does not hold true for the auditory input. Stimulus frequency, and not "space," is repre- 
sented systematically across the sensory epithelium of the cochlea. Though the properties of the auditory input have yet to be studied thoroughly, preliminary investigations reveal no hint of a topographic (i.e., tonotopic) projection (Mast and Chung, 1973; Chalupa and Rhoades, 1977; Allon and Wollberg, 1978). Instead, auditory responses show a subtle but definite dependence on the location of the sound source in space. Moreover, the directions in the horizontal plane to which units respond change along the anterior-posterior axis of the tectum (Wickelgren, 1971; Gordon, 1973; Dräger and Hubel, 1975; Chalupa and Rhoades, 1977; Harris et al., 1980). If these responses reflect a "space-dependent" organization of the auditory input, they would indicate that projections to the tectum are determined by the sensory space that they represent rather than by the topographies of the sensory epithelia per se.

The existence of a space-dependent auditory projection has not been established rigorously because of an apparent lack of neuronal selectivity for sound location; the reason for this lack of selectivity is not clear. Perhaps auditory neurons in the tectum are poorly tuned in space; perhaps accurate and appropriate positioning of the pinnae is essential for strong, space-dependent responses; or possibly, the reverberant acoustic conditions and imprecise methods of sound presentation used in the previous studies obscured the spatial sensitivity of the tectal neurons. That auditory neurons can exhibit considerable spatial selectivity is demonstrated by the responses of a special class of neurons called limited field neurons that occur in the midbrain and forebrain of the owl (Knudsen et al., 1977; Knudsen and Konishi, 1978a, b). These neurons are excited only by sounds originating from within a restricted region of space, or receptive field, and are inhibited by sounds originating outside of this region (Knudsen and Konishi, 1978c). In the midbrain nucleus MLD (mesencephalicus lateralis pars dorsalis), which is homologous to the inferior colliculus, limited field neurons are organized systematically according to the locations of their receptive fields so that they form a physiological map of auditory space. These neurons were studied under anechoic conditions, with precisely controlled stimuli, in an animal with immobile external ears.

The barn owl is an ideal subject for investigating the questions of whether auditory neurons in the optic tectum are tuned in space and, if so, whether the neurons are organized according to their spatial tuning. As mentioned previously, the degree to which a sensory modality is represented in the tectum seems to increase with the importance of the modality in guiding the animal's orientation behaviors. In barn owls, the auditory system plays a crucial role in prey capture: since these birds hunt at night when visual cues may be of little or no value, they rely heavily on their highly developed auditory systems to localize the sounds of their prey and to guide at least the initial stages (and potentially all) of their attack (Payne, 1971; Konishi, 1973). Hence, a strong auditory input to the tectum might be expected. In addition, the barn owl localizes sounds with extreme accuracy both in azimuth and elevation (Knudsen et al., 1979), a capacity that has correlates in the receptive field properties of central auditory neurons (Knudsen and
Konishi, 1978a). Furthermore, since neither the ears nor the eyes of the owl move independently of its head, fixing the head during recording experiments assures that the ears and eyes remain in stationary and normal positions. This latter attribute is particularly advantageous when investigating the alignment of auditory and visual receptive fields of single neurons.

The findings presented here demonstrate that the auditory projection to the owl's optic tectum is more pervasive than has been reported for any extravisual input in any other species. Neurons in the tectum have well defined auditory receptive fields and are organized according to the locations of their fields, giving rise to a two-dimensional map of auditory space across the tectum. Finally, the maps of auditory and visual space were found to be remarkably similar and closely aligned, suggesting that the projections of sensory modalities to the tectum are determined by the sensory space that they represent and not by the topographies of their sensory epithelia.

\section{Materials and Methods}

Experimental conditions. Experiments were performed in a sound isolation chamber (IAC 404A) fitted with interior fiber glass panels to suppress echoes. Free field conditions existed in the central portion of the room for frequencies between 1.25 and $16.0 \mathrm{kHz}$. Deviations from a free field were detected at $1.25 \mathrm{kHz}$ and these increased with decreasing frequency.

Acoustic stimuli were generated with a $4-\mathrm{cm}$ dynamic speaker mounted on a narrow semicircular track (Fig. 1). The location of the speaker was under remote electronic control. The speaker moved in azimuth by traveling along the track and in elevation by moving with the track as it pivoted around a horizontal axis. The radius of the track was $92 \mathrm{~cm}$. With the owl at the center of this apparatus, the speaker could be positioned nearly anywhere around the owl with an accuracy of $\pm 0.2^{\circ}$ in azimuth and $\pm 0.5^{\circ}$ in elevation. The controls and monitors of the speaker's location were outside of the sound chamber.

Sound stimuli consisted of noise and tone bursts which, unless otherwise stated, were $100 \mathrm{msec}$ in duration with 2.5-msec rise and fall times presented once per sec. Sound levels, given in decibels relative to threshold, were controlled with a decade attenuator. The frequency response of the system was calibrated with the $1 / 2$-inch microphone placed at the center of the chamber where the owl would be located. Sound pressure levels were constant $( \pm 3 \mathrm{~dB})$ from 2 to $12.5 \mathrm{kHz}$ and decreased at a rate of $20 \mathrm{~dB}$ per octave below $2 \mathrm{kHz}$ down to $630 \mathrm{~Hz}$. The spectrum of the noise stimulus was shaped by these filter properties. When measurements involved tonal stimuli, the filter properties of the system were corrected by compensatory amplification.

Visual stimuli were rear-projected onto an opaque Plexiglas hemisphere that was wheeled into the sound chamber and locked into place in front of the owl. The radius of the hemisphere was $57 \mathrm{~cm}$, and the owl's head was located at its center. Spots and bars of various lengths and widths, of various speeds and orientations, and of positive and negative contrasts were presented 


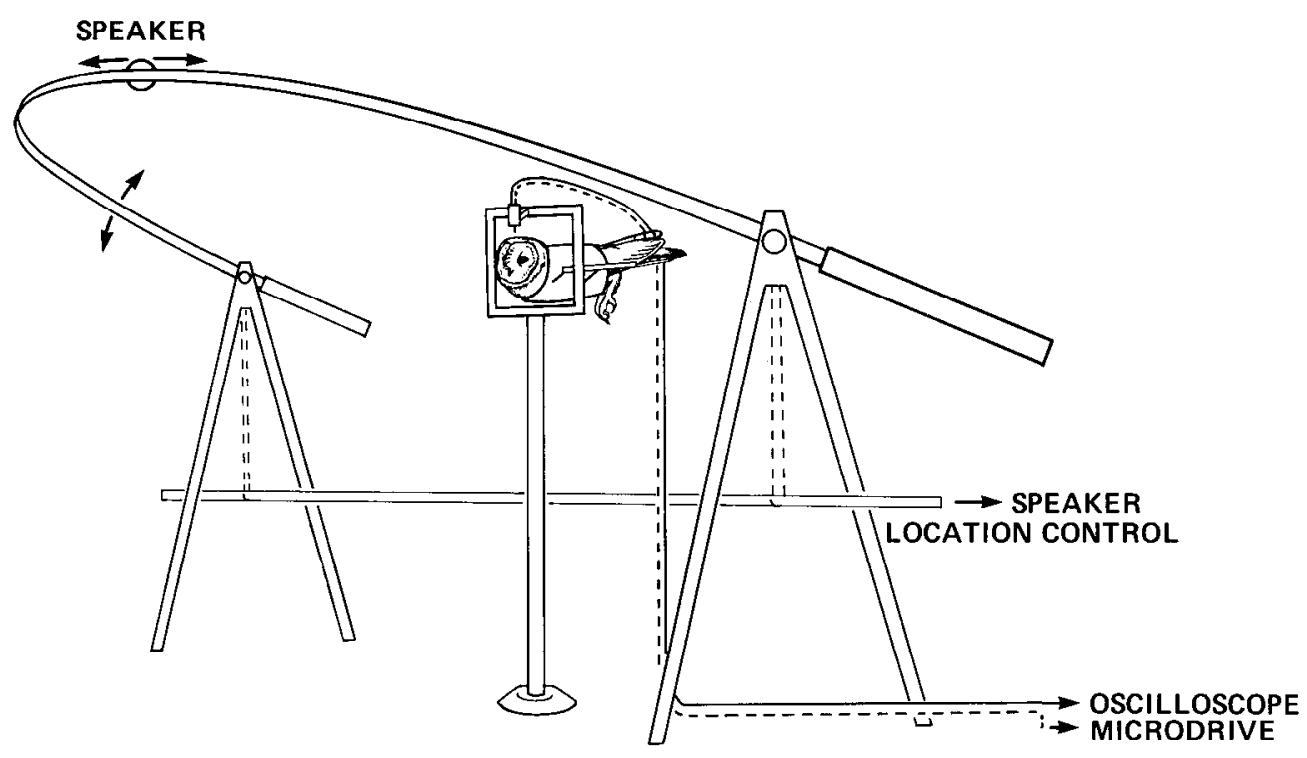

Figure 1. The experimental setup. The owl is suspended in a prone position with its head bolted to the stereotaxic frame and positioned in the center of the speaker-carriage system. The speaker moves along the semicircular track, and the track itself pivots around the horizontal axis. This equipment is housed inside a sound isolation chamber with the controls for speaker position located outside of the chamber. The opaque hemisphere, which was wheeled into the chamber for visual testing, is not shown.

using a hand-held projector. Visual receptive fields were mapped directly on the hemisphere. When the hemisphere was not being used, it was removed from the chamber.

Head and eye positions. The cars and cyes of the barn owl are virtually immobile in the head (Payne, 1971; Pettigrew and Konishi, 1976). Eye movements, monitored ophthalmoscopically with the head fixed, consisted of occasional drifts or rapid saccades, after which the eyes would return to a standard resting position. The largest movements were $2^{\circ}$ saccades induced by startling the owl with a loud clap. In a few cases, the eyes diverged slightly $\left(<1^{\circ}\right)$ just after anesthesia was induced. However, this divergence disappeared within 5 to $10 \mathrm{~min}$. These characteristics are similar to those observed in the burrowing owl (Cooper and Pettigrew, 1979).

Because of the immobiiity of the eyes, retinal landmarks provide a convenient and accurate means for positioning the head (and ears) precisely in the sound chamber. The landmark used was a thin strip of pigmented tissue, called the pecten oculi, that overlies the optic nerve head. The projection into space of the superior limb of the pecten is $8^{\circ}$ above the visual axis of the eye (Pettigrew, 1979). The visual axis is the line projecting from the center of the area centralis (barn owls do not have foveae) through the nodal point of the eye to the horizon.

To orient the head (and therefore the eyes and ears) in the chamber, the axis connecting the ear canals (interaural axis, Fig. 2) was aligned with the axis of rotation of the speaker track (Fig. 1). The head then was rotated about the interaural axis until the superior limbs of the pecten were at $+8^{\circ}$ in elevation according to the speakermoving apparatus. In this position, the horizontal meridian of the eyes was horizontal with respect to the ground and coincident with $0^{\circ}$ elevation of the speaker. The midsagittal plane of the head was aligned with $0^{\circ}$ azimuth of the speaker by rotating the head about its vertical axis (Fig. 2) until the projections of the pecten were left-right symmetrical.

Auditory and visual coordinate systems. Due to the design of the speaker carriage apparatus, the speaker moved in a horizontal polar coordinate system. During experiments, speaker locations were recorded using this coordinate system. Visual fields were mapped using the same coordinate scheme. A horizontal polar grid was etched into the visual hemisphere. When the hemisphere was locked into place in front of the owl, the azimuth and elevation coordinates marked on the hemisphere lined up with the same coordinates defined by the speaker with an accuracy of better than $1^{\circ}$ for nearly all directions.

A major disadvantage of the horizontal polar coordinate system is that the distance on a unit sphere corresponding to $1^{\circ}$ in elevation varies with azimuth. For example, a $1^{\circ}$ change in elevation at azimuth $0^{\circ}$ (straight ahead) corresponds to more than 6 times the distance on a unit sphere as a $1^{\circ}$ change in elevation at azimuth $80^{\circ}$. If one wishes to compare receptive field sizes in angular terms over a wide range of azimuths, it is imperative that $1^{\circ}$ of angle correspond to a standard distance on a unit sphere. This is accomplished by converting horizontal polar coordinates into a double pole coordinate system in which azimuth and elevation are defined by independent axes. The mutual independence of the axes results in a constant relationship between angular deviation and distance on a unit sphere.

The double pole coordinate system used in this study employs as reference axes the owls' interaural axis and vertical axis (the line passing vertically through the center of the head when the visual axes are horizontal (Fig. $2 B)$ ). The interaural and vertical axes establish three 
(A)

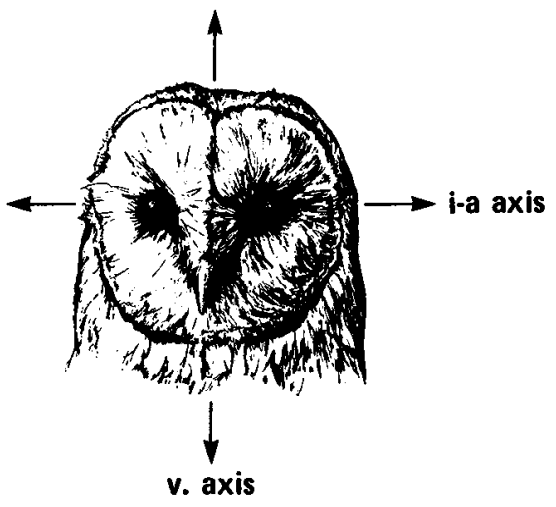

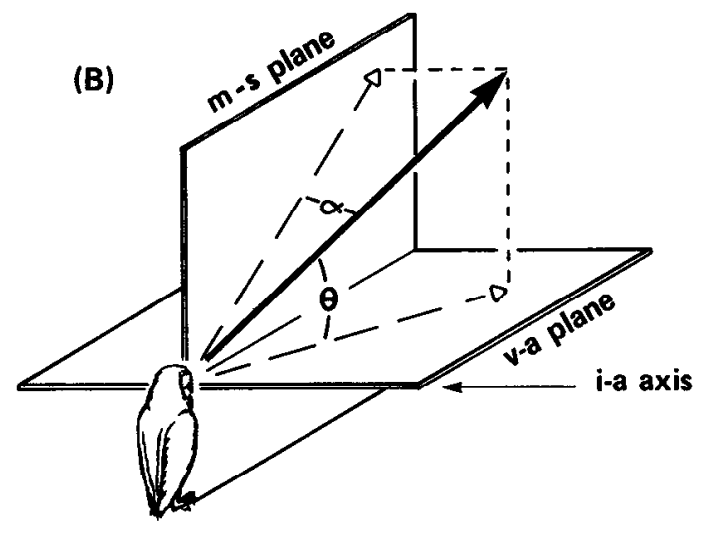

(C)

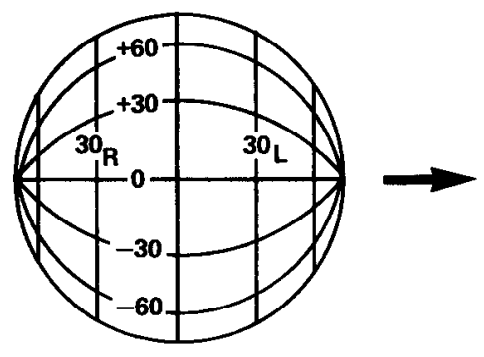

POLAR

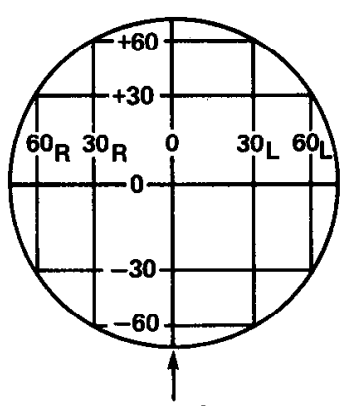

v. axis

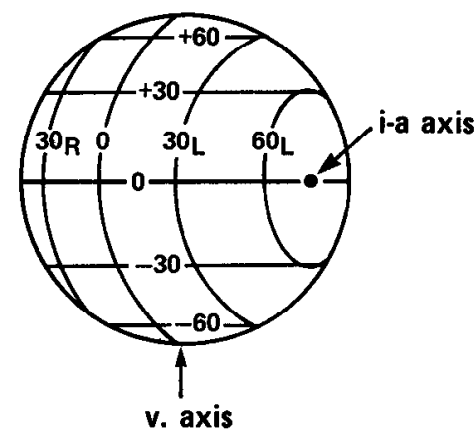

v. axis

DOUBLE POLE

Figure 2. Definition of spatial axes and the double pole coordinate system. $A$, Double pole coordinates are defined with respect to the owl's interaural (i-a axis) and vertical (v.axis) axes. The interaural axis passes through the centers of the ear canals; the vertical axis passes vertically through the center of the head when the owl's visual axes are parallel to the ground. $B$, In this double pole space, azimuth $(\alpha)$ is the angular deviation left $(L)$ or right $(R)$ from the midsagittal plane (m-s plane); elevation $(\theta)$ is the angular deviation above $(+)$ or below $(-)$ the visuoaural plane ( $v$-a plane). $C$, The speaker (Fig. 1$)$ moved according to a horizontal polar system (left). Speaker coordinates were converted mathematically into double pole coordinates (right).

reference planes: The midsagittal, visuoaural, and transverse planes. The midsagittal plane contains the vertical axis and is orthogonal to the interaural axis. The visuoaural plane contains the visual axes of the eyes and the interaural axis and is orthogonal to the vertical axis. The transverse plane contains both the interaural and vertical axes. During experiments, the owl's head was bolted to the stereotaxic frame so that the midsagittal and transverse planes were vertical and the visuoaural plane was horizontal.

The origin $\left(0^{\circ}\right.$ azimuth, $0^{\circ}$ elevation) of this coordinate system is the ray formed by the intersection of the midsagittal and visuoaural planes that starts at the center of the head and projects forward, directly in front of the owl. Azimuth is defined as the angular deviation from the owl's midsagittal plane in degrees left (L) or right (R). Elevation is defined as the angular deviation from the owl's visuoaural plane in degrees up (+) or down (-). When viewed from the origin, the contour lines of isoazimuth and isoelevation appear as perpendicular sets of parallel lines. When projected onto a sphere, they form orthogonal sets of circles (Fig. 2C).

To avoid front-back ambiguity in notation, azimuthal and elevational coordinates are changed as they cross the transverse plane from angular values of $x^{\circ}$ to the complementary value of $\left(180^{\circ}-x^{\circ}\right)$. Thus, angles of less than $90^{\circ}$ refer to locations in front of the owl, while angles from $90^{\circ}$ to $180^{\circ}$ signify directions behind the owl. With this convention, an isoazimuth or isoelevation contour on a sphere is a hemicircle that terminates at the transverse plane.

In this double pole coordinate system, an azimuth of $90^{\circ}$ defines a direction in line with the interaural axis. Since this axis lies in the visuoaural plane, the elevation of the direction must be $0^{\circ}$. As azimuth $(\alpha)$ decreases from $90^{\circ}$, the range of possible elevations increases by $\pm\left(90^{\circ}-\alpha\right)$ so that a full range of $\pm 90^{\circ}$ in elevation at a single azimuth occurs only when $\alpha=0^{\circ}$ (i.e., in the midsagittal plane). The same logic holds true in elevation: the only elevation at which all azimuths are possible is $0^{\circ}$. An elevation of $90^{\circ}$ means that the direction is straight up or down and therefore must be at $0^{\circ}$ azimuth. Notice that, in this coordinate space, azimuth and elevation are orthogonal only in the midsagittal and visuoaural planes.

During the experiments, measurements of acoustic and visual stimulus locations were made in horizontal polar coordinates. Afterwards, these values were converted into double pole coordinates. Azimuth values required no conversion, since horizontal polar azimuth and double 
pole azimuth are equivalent (Fig. 2). Polar elevation was converted into double pole ( $\mathrm{EL}_{\mathrm{dp}}$ coordinates by canceling the azimuthal (az) contribution to the polar value by applying the formula $E L_{d p}=\sin ^{-1}[\sin$ el $\cdot \cos$ az]. All data analyses and descriptions are based on these derived double pole values.

The preparation. Recordings were made from the left and right tecta of eight barn owls (Tyto alba). Each owl was anesthetized with ketamine hydrochloride $(25 \mathrm{mg}$ / $\mathrm{kg}$ ) and a stainless steel well was inserted through a trephined hole in the skull over the left tectum. A small steel plate with protruding bolts also was cemented to the skull to provide an anchor for fixing the head during the recording sessions. Prepared in this manner, recordings could be made from each owl in numerous experiments over a period of weeks.

On the day of an experiment, anesthesia was induced with ketamine hydrochloride injected intramuscularly and was maintained at a light level with repeated injections. The owl was wrapped in a soft leather harness and suspended by springs from a stereotaxic frame in prone position. The head was centered and aligned in the speaker-moving apparatus (as described above) and bolted rigidly to the stereotaxic frame. The electrode carriage system and stereotaxic frame (Fig. 1) were designed to minimize sound field distortions. Tests run with this equipment in place revealed no measurable distortions at the site where the head would be located for frequencies up to $8 \mathrm{kHz}$.

Paralysis was unnecessary because of the natural immobility of the eyes. The eyelids were relaxed with a drop of phenylephrine hydrochloride and retracted. Refractive error was typically -2 diopters and never more than -3 diopters at $57 \mathrm{~cm}$ (the distance to the visual hemisphere). In early experiments, contact lenses were applied to prevent corneal clouding. However, under the light levels of anesthesia used, the nictitating membranes continued to sweep periodically across the eyes and the corneas remained clear without lenses. Hence, the use of lenses was discontinued.

Recording and analysis. Tectal units were recorded extracellularly with glass micropipettes filled with $3 \mathbf{M}$ $\mathrm{NaCl}$ and glass-coated platinum/iridium electrodes. Electrodes were positioned under visual guidance using a rack and pinion carriage system capable of three-dimensional movement. Once in position, the electrode was advanced into the brain with a Kopf hydraulic microdrive, the controls of which were located outside of the chamber.

Nerve impulses were passed through a high impedance follower located just behind the owl, and the buffered signal was conducted out of the chamber for amplification and display. Unit activity was monitored visually on a storage oscilloscope and acoustically with an audio monitor. A level discriminator and event counter enabled on-line analysis of thresholds, best areas, best frequencies, and intensity response profiles. In addition, the responses of selected units were recorded on magnetic tape for off-line computer analysis.

The search stimulus. As the electrode approached the tectal surface, an acoustic search stimulus was switched on to drive the units. The stimulus was the standard noise burst presented once per sec at a sound level of 30 to $40 \mathrm{~dB}(\mathrm{~A})$. Whenever a unit was encountered, whether driven by the stimulus or not, its responsiveness to light and sound was tested. It is important to note, however, that the search stimulus selected for acoustically responsive, nonhabituating units.

Histology and mapping procedures. All but the final experiment on each owl were conducted on the left tectum. In the last experiment on the left side, the surface of the brain was photographed and a series of four to eight electrode penetrations was made in a grid pattern. Lesions were placed at the sites of the first and last tectal units in each penetration. In a final experiment, a second recording chamber was implanted on the right side, units were recorded, and horseradish peroxidase was injected (to be reported in a later paper). After 2 days of survival, the owl was anesthetized deeply with sodium thiopental and perfused through the heart with $1 \%$ paraformaldehyde and $2 \%$ glutaraldehyde in $5 \%$ sucrose. The head was remounted in the stereotaxic frame and the brain was blocked in the transverse plane, parallel to the electrode tracks. Transverse sections were cut $30 \mu \mathrm{m}$ thick on a freezing microtome. Every third section was mounted and stained with cresyl violet.

Top and bottom lesions were identified and correlated with specific electrode tracks. The positions of units encountered between the lesion sites were reconstructed from their depths relative to the lesions as measured from the microdrive.

Lesions and reconstructed units from the left tecta of all of the brains were recorded in a single atlas consisting of 40 photomicrographs taken at $180-\mu \mathrm{m}$ intervals through the left tectum. For the purposes of mapping, the tectum was treated as a two-dimensional, curved sheet of cells (Fig. 3). The anteroposterior dimensions ( $y$ dimension) was divided by the atlas into 40 subdivisions, representing intervals of $2.5 \%$. Each subdivision was represented by a line, the length of which corresponded to the circumferential extent ( $x$ dimension) of the tectal subdivision. To collapse the thickness of the tectum into a single plane, the circumferential length of each layer was scaled up to that of the stratum opticum (layer 1, Fig. 4). This technique assumed that the response topographies in the shorter inner layers were uniform and linear compressions of the topography in the outer layer. This approach, chosen for its simplicity, draws some validity from the radial patterns of neural processes that traverse the layers (Fig. 4). However, local inhomogeneities of representation may well exist within individual layers, and these will have contributed variability to values along the $x$ dimension.

The 40 section lines, representing the flattened tectal surface, were aligned with respect to each other by their midpoints so that units located $50 \%$ of the way around lined up along the $x$ dimension (Fig. 3). This method of aligning the sectional lines distorted the topography less than did aligning the medial or lateral edges of the lines. Nevertheless, distortions did occur and these must be kept in mind when interpreting the physiological maps. The nature of the distortion is an apparent expansion of representation caused by stretching the distance between contiguous points on adjacent sections. These distortions 

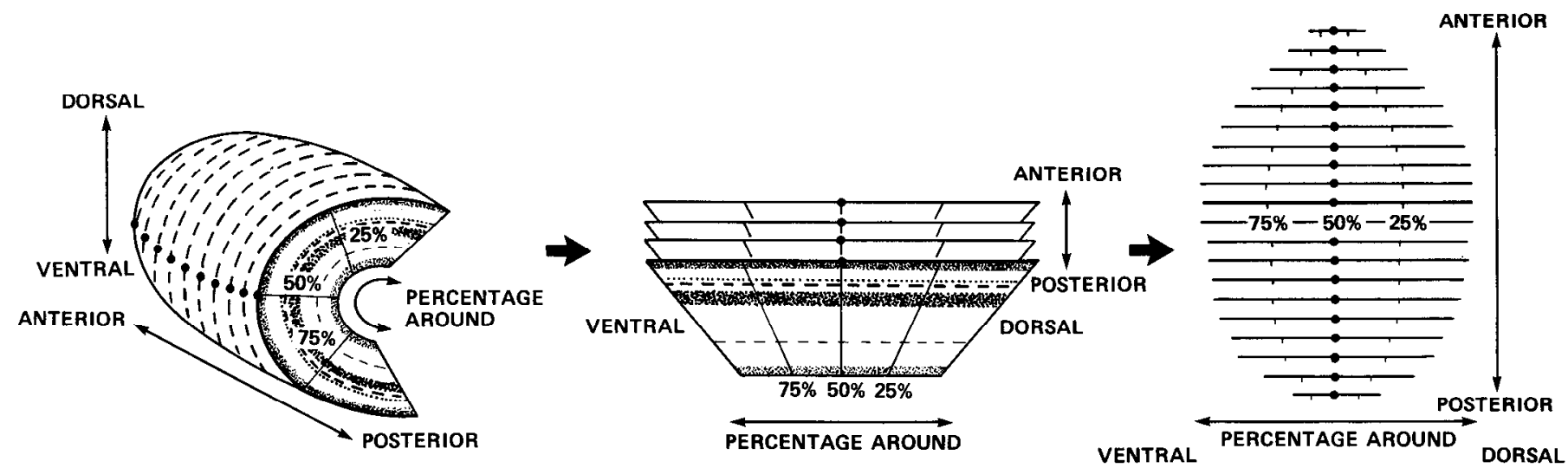

Figure 3. Flattening the optic tectum. To create maps of functional topography (Figs. 8 and 12) and to determine magnification factors (Figs. 9 and 13), units were located in a two-dimensional projection of the tectal surface based on their distances from the anterior pole and their circumferential distances from the dorsomedial edge. The tectum was sectioned in the transverse plane. The distance of a unit from the anterior pole was based on the number of sections to the anterior pole expressed as a fraction of the total number of tectal sections $(\times 100=$ percent). Determining a unit's distance from the dorsomedial edge was more tedious because the tectum is a thick, curved slab of cells in which the circumferential distance along the inner surface is substantially shorter than along the outer surface. Therefore, distances around were normalized in a layer-specific manner by expressing the location of a unit as a percentage around in ils respective cell layer. Metric distances were recovered by treating the circumference of the outermost layer (stratum opticum) and the anteroposterior length of the tectum as $100 \%$ values.

occur at the anterior and posterior ends of the map, wherever the width of the tectum ( $x$ dimension) changes along its length. The magnitude of the distortion increases with the rate of change in width and is greatest near the edges of the map. Where tectal width is constant, over the middle $50 \%$, no distortion from section line alignment occurs.

Nomenclature for layers of the optic tectum. The optic tectum was divided into 15 layers based on the system of Ramon y Cajal (1972). For this paper, we need only distinguish between neurons located above or below layer 11 (Fig. 4). Layers 1 to 10 will be referred to as the "superficial layers" (strata griseum et fibrosum superficiale of Cowan et al. (1961)) and layers 11 to 15 will be referred to as the "deep layers."

\section{Results}

The extent of the visual field. The visual fields of the left and right eyes were measured in two awake, restrained birds. The measurements were made by viewing a retina ophthalmoscopically at a distance of $1 \mathrm{~m}$ and noting the coordinates of the locations at which the retina became occluded by the iris (tcmporal, inferior, and superior limits) or obstructed by the lore feathers that extend from the center of the face (nasal limit). At this distance and with the head secured in standard position, the visual axes of the eyes were at $0^{\circ}$ elevation and about $1^{\circ}$ ipsilateral of $0^{\circ}$ azimuth (Pettigrew, 1979).

The left and right eyes viewed equally large, mirror symmetrical regions of space (Fig. 5). Along the horizontal meridian, the field extended from $95^{\circ}$ ipsilateral to $21^{\circ}$ contralateral; along the vertical meridian, it extended from $+45^{\circ}$ to $-50^{\circ}$. The vertical extent of the visual field increased temporarily and was greatest at $20^{\circ}$ ipsilateral where it ranged from $+55^{\circ}$ to $-50^{\circ}$. More temporally, the lower limit ascended, causing the vertical range to decrease.

The area of binocular overlap was the frontal $42^{\circ}$ in azimuth and from $+45^{\circ}$ to $-50^{\circ}$ in elevation. The area was approximately teardrop shaped, with the area below $0^{\circ}$ in elevation making up 58\% of the total binocular field.

Unit activity associated with tectal layers. Three types of spike activity were recorded in the tectum: hash, bursty units, and regularly discharging units. Hash was low amplitude, high frequency activity that did not resolve into unit activity. Bursty units often discharged two or more spikes in rapid succession (1- to 4-msec intervals) and then paused before discharging in another burst (Fig. 4). Regular units discharged spikes at relatively even intervals. Each type of activity was associated with particular cell layers in the tectum.

Hash was recorded in the most superficial and deep layers. In the superficial layers, the hash responded to visual stimulation of the contralateral eye only and not to acoustic stimuli. The visual field was always small (usually less than a few degrees across) and sharply defined. Moreover, the hash would respond consistently to fast repetitions of high velocity stimuli that were not effective in driving nearby units. These response properties are similar to those of retinal ganglion cells, which are known to project directly to the first seven superficial layers of the tectum (Ramon y Cajal, 1972; Cowan et al., 1961).

In the deep layers, the hash responded either to sound alone or to sound and light. The auditory responses were selective for sound location with a spatial tuning that matched that of single units recorded in the vicinity. Similarly, the visual responses, when present, exhibited receptive fields at the same locations as those of nearby units. This hash activity probably is associated mainly with afferent auditory fibers and efferent bimodal fibers that traverse the deep layers.

Bursty units were recorded in superficial layers 2 through 10 and never in the deep layers (23 reconstructed recording sites). The bursting discharge pattern was prevalent in both heavily and very lightly anesthetized 


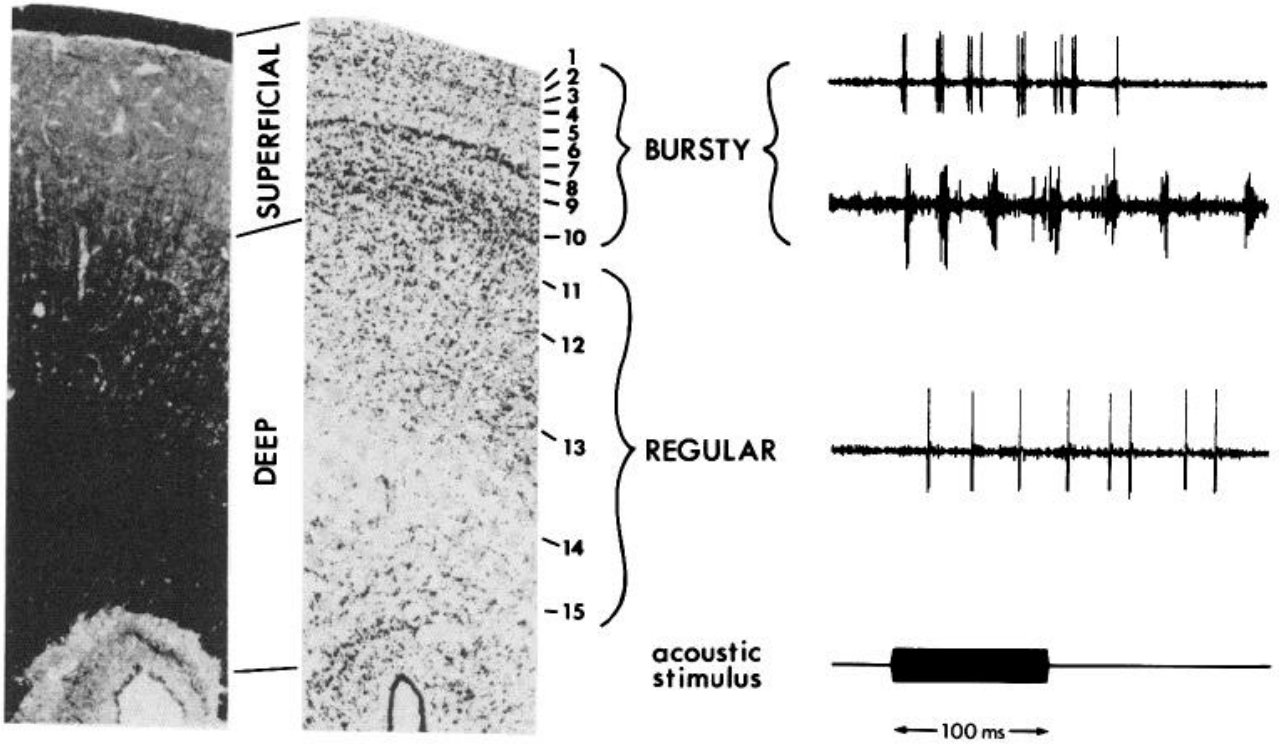

Figure 4. Cell layers in the optic tectum and the types of unit activity recorded in them. To the left are histological strips from transverse sections taken midway along the length of the tectum showing the 15 cell layers of the optic tectum. The strip on the far left was stained for myelin using the Weigert technique; the strip on the right was stained for cell bodies using a Nissl stain. The layers were numbered according to the system of Ramon y Cajal (1972). The superficial layers are defined as layers 1 through 10 and correspond to the stratum opticum and the strata griseum et fibrosum superficiale of Cowan et al. (1961). Notice that, with the exception of the stratum opticum, these layers are relatively free of myelinated fibers. To the right are shown unit responses to a noise burst stimulus. These recordings demonstrate the differences between the activity recorded in the superficial and deep cell layers: units in the superficial layers are difficult to isolate and respond with bursts of spikes that occur in synchrony with bursts from neighboring cells. Units in the deep layers are isolated easily and respond to the acoustic stimulus with regularly spaced spikes.

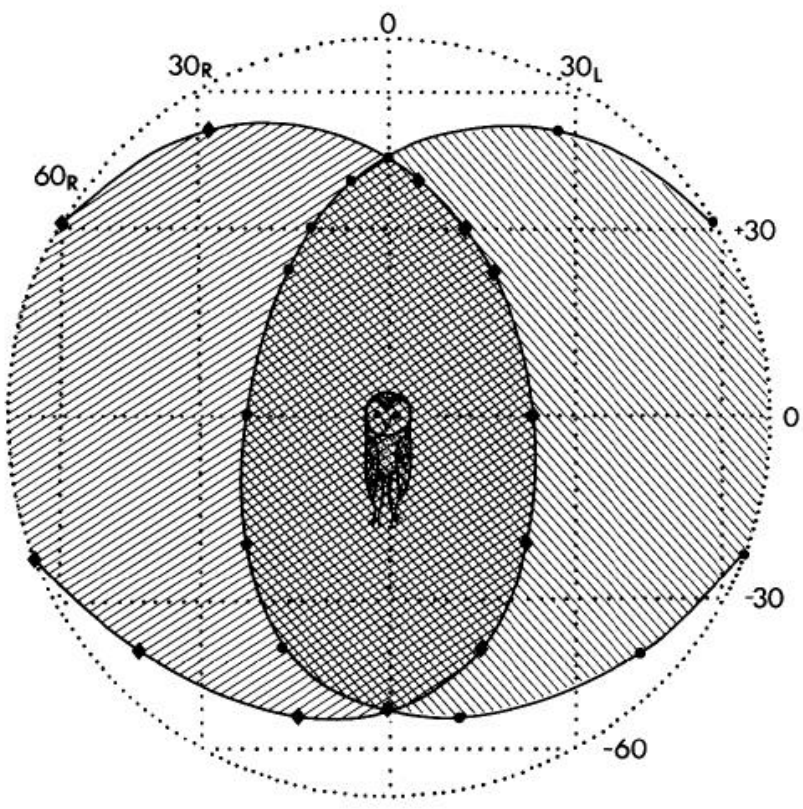

Figure 5. The visual field of the barn owl. The visual limits of the left eye (๑) and right eye $(\bullet)$ are projected onto a grid in double pole coordinates.

birds and therefore is not likely to be a consequence of the anesthetic. Bursty units were difficult to isolate, and only 5 out of 87 recordings were clearly from single units.

The waveforms of bursty units were bi- or triphasic and about $1 \mathrm{msec}$ in duration, suggesting that they were soma spikes of resident neurons. In multiunit recordings, the units tended to discharge in concert even though the timing of the bursts was irregular (Fig. 4), as though the units were mutually coupled or driven by a common source. A finding also shown in Figure 4 is that the time between bursts was similar to the time between spikes of regular units recorded in the deeper layers. The possible significance of this correspondence is not yet clear.

Bursty activity often persisted at a low level without stimulation and increased dramatically in response to either visual or acoustic stimuli. The same units seemed to respond to both stimulus modalities; however, this can be stated with certainty only for the 5 units that were isolated. When stimulated with sound, bursty units responded at defined latencies and continued to respond throughout, and often well beyond, the duration of the stimulus (Fig. 4). Minimum response latencies, measured from the time when sound entered the ear canal, ranged from 10 to $21 \mathrm{msec}$ with a median of $13 \mathrm{msec}(n=37)$.

Also encountered in the superficial layers were units with regular discharges. These responded only to visual stimuli and were not studied in detail.

In the deep layers, only regularly discharging units were recorded. Out of 79 such units, 70 were bimodal, 9 responded only to sound, and none responded only to light. These large, biphasic spikes typically had resting discharge rates below $5 / \mathrm{sec}$ and some below $1 / \mathrm{sec}$. When the speaker was at an appropriate location, $80 \%(63 / 79)$ responded in some sort of sustained fashion to a noise 


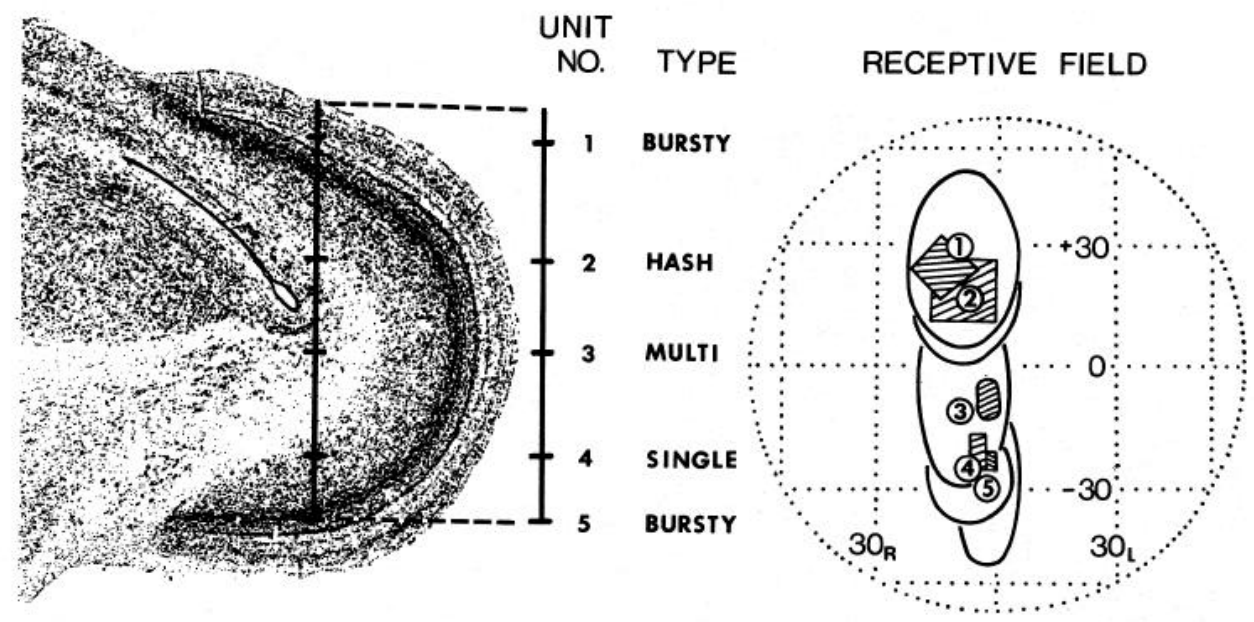

Figure 6. An electrode track reconstruction showing the types of unit activity and the visual and auditory receptive fields associated with each recording location. On the left is a Nissl-stained, transverse section through the left optic tectum. The numbered ticks along the line indicate the depths at which unit activity was recorded. The various types of activity are defined under "Results." All of the recordings were from bimodally sensitive units. On the right are drawn the visual (hatched areas) and auditory (bold lined oblongs) receptive fields. The encircled numbers, which correspond to the unit numbers, are located at the centers of the auditory best areas. The visual fields, auditory fields, and best areas all descended systematically as the electrode penetrated dorsoventrally.

burst stimulus. The remaining $20 \%$ responded only phasically regardless of stimulus location. Of the units with sustained responses, about $35 \%$ continued to discharge after the stimulus had ended (Fig. 4). Minimum response latencies of regular units to sound ranged from 6 to 77 msec with a median of $13 \mathrm{msec}(n=51)$.

The following descriptions of response properties are based on data from 187 recording sites: 87 from bursty units in the superficial layers, 79 from regular single units in the deep layers, and 21 from multiunit recordings of regular unit activity in the deep layers. When it is necessary to distinguish among these types of units, they will be referred to as "bursty units" (implying a multiunit recording), "regular units," and "regular multiunits."

Visual receptive fields of bimodal units. Nearly all units encountered responded to visual stimulation. Bursty units (layers 2 through 10 ) responded strongly and continuously to slowly moving visual stimuli in their receptive fields. Excitatory fields ranged in size from $2^{\circ}$ to $30^{\circ}$ in azimuth and from $2^{\circ}$ to $23^{\circ}$ in elevation (inhibitory areas were not investigated). Some units preferred either positive or negative contrasts, but most responded equally well to either. Out of 20 recordings from bursty units with receptive fields in the binocular region, 10 showed no evidence of excitatory input from the ipsilateral eye. In the remaining recordings, an ipsilateral excitatory effect was weak but definitely present.

The visual response properties of regular units (deep layers) were quite variable. Some were excited strongly by visual stimuli, while others barely responded at all. Excitatory field sizes ranged from $1^{\circ}$ to $45^{\circ}$ in azimuth and from $1^{\circ}$ to $32^{\circ}$ in elevation. Often found within the larger fields was a much smaller area to which the unit responded most strongly. Nearly half ( 22 of 50$)$ clearly preferred either positive (11) or negative (11) contrast. Most preferred bars to spots, and some required the bar to be in a particular orientation. All regular units with fields in the binocular region were influenced to some extent by the ipsilateral eye, although the contralateral eye was always dominant. Out of 20 units, the ipsilateral eye drove 8 well and 6 weakly and facilitated responses to contralateral eye stimulation in 6 .

Visual topography. The visual receptive fields of bursty and regular units, which were aligned radially in the tectum, were superimposed in space (for example, units 4 and 5 in Fig. 6 and the medial penetration in Fig. 7). Therefore, the data from bursty and regular unit recordings were combined to derive the topography of the visual representation of space in the tectum. Each unit was assigned a single pair of spatial coordinates based on the geometric center of its field; the location of the unit in the tectum was reconstructed as described in Figure 3. Lesions were placed and recovered histologically for 27 penetrations in eight birds. The field coordinates for the 63 units reconstructed from these penetrations were entered on a flattened projection of the tectal surface (Fig. $3)$. Locations representing the same azimuth or elevation were connected by lines and finally, these lines were smoothed to give the contours of isoazimuth and isoelevation illustrated in Figure 8.

The entire visual field of the contralateral eye projected topographically onto the tectum. Receptive field centers ranged in azimuth from $18^{\circ}$ ipsilateral at the anterior pole to $70^{\circ}$ contralateral at the caudal pole and ranged in elevation from $+42^{\circ}$ dorsomedially to $-46^{\circ}$ ventrally. The horizontal meridian $\left(0^{\circ}\right.$ elevation) was represented anteroposteriorly at about $50 \%$ around dorsoventrally (Fig. 8). The vertical meridian $\left(0^{\circ}\right.$ azimuth) ran dorsoventrally with a slight anterior inclination in the dorsal tectum and a sharp curve toward the posterior pole in the ventral tectum. Isoazimuth contours followed similar trajectories. As a consequence, dorsoventral electrode penetrations recorded progressions of receptive fields that moved forward as well as downward in space as the electrode advanced through the ventral half of the tectum (Figs. 6 and 7). The highest and lowest fields were 


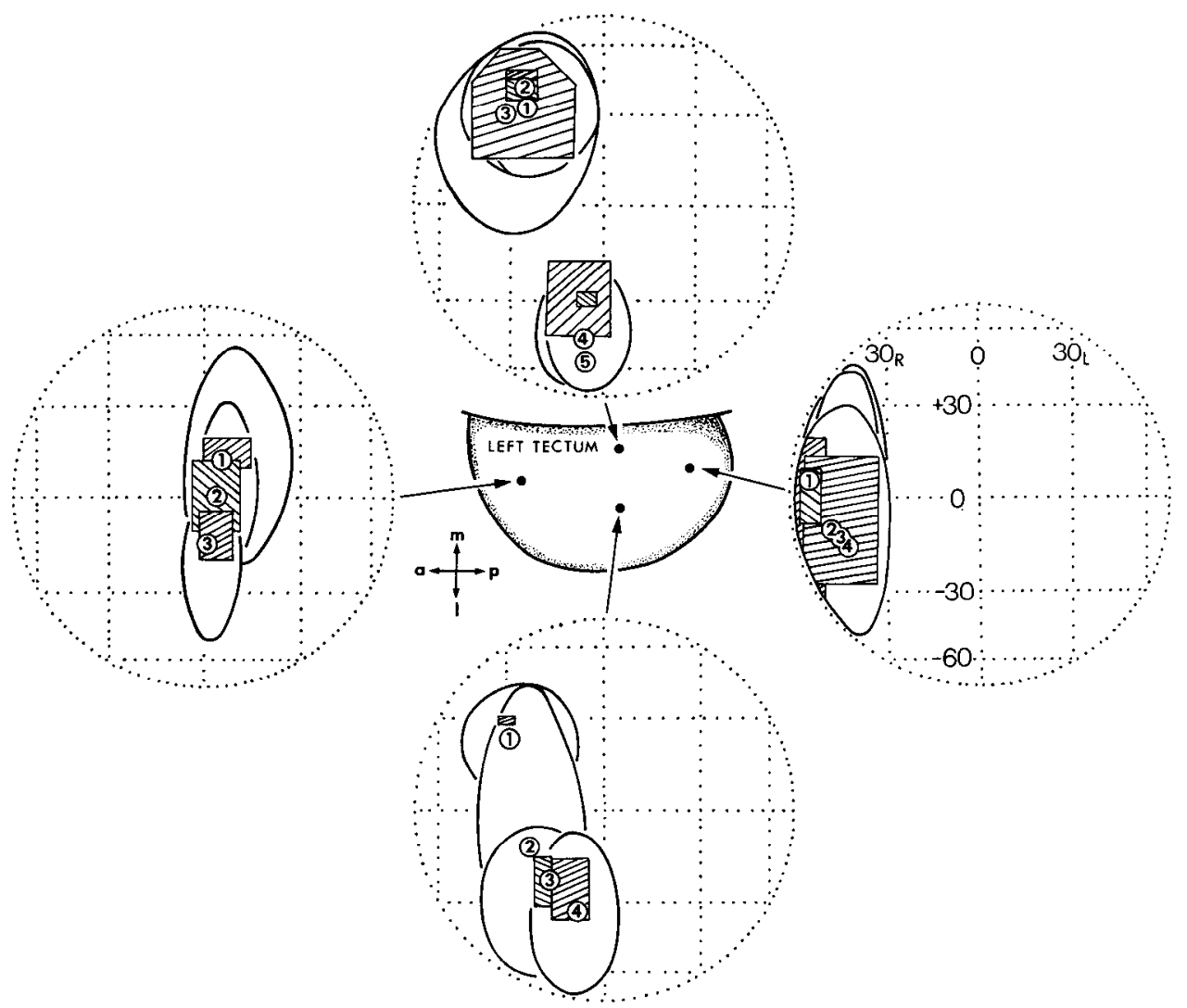

Figure 7. Visual and auditory receptive fields of sequentially recorded, bimodal units from four separate electrode penetrations in the left optic tectum. The penetrations were made at the locations indicated by the solid circles on the dorsal view of the tectum (center). The visual receptive fields are hatched; the auditory receptive fields are oblongs; the centers of auditory best areas are numbered. The numbers represent the order in which the units were encountered during each dorsoventral penetration. The receptive fields from the most medial penetration (top center) jumped from high to low. This is because the tectum curves around underneath (Fig. 6), causing such medial penetrations to intersect both the dorsomedial (high fields) and ventromedial (low fields) edges of the tectum at two discontinuous portions of the track. $a$, Anterior; $p$, posterior; $m$, medial; $l$, lateral.

located dorsomedially and ventrally, about 60 to $70 \%$ of the distance back from the anterior pole.

Because the tectum is a curved slab of cells that partially enwraps the midbrain auditory nucleus MLD (Fig. 6), penetrations made at the medial edge intersected the tectum twice. Such penetrations encountered units with extremely high fields, followed by unresponsive tissue or purely auditory units in the MLD, and finally more tectal units with very low fields (Fig. 7). This shift in field elevation became less abrupt in penetrations made more laterally. At the lateral edge, where penetrations were short and tangential to the tectal surface, the fields clustered at the horizontal plane.

Based on area measurements made on the tectal map (Fig. 8), approximately $60 \%$ of the tectum is devoted to the zone of binocular overlap (Fig. 3), which constitutes only $35 \%$ of the visual field of the contralateral eye. The greatest representation is given to directions within $10^{\circ}$ of the visual axis which account for $5 \%$ of the eye's visual field but occupy $15 \%$ of the tectal map.

Magnification factors (micrometers along the stratum opticum per degree of visual space) for azimuth and elevation were estimated by plotting the coordinates of receptive field centers versus the locations of the units in the tectal projection (Fig. 9): field azimuth versus unit distance from the anterior end of the tectum (Fig. 9A) and field elevation versus unit distance from the dorsomedial edge (Fig. 9B). The slopes of the regression lines calculated for these point scatters represent average magnification factors along the surface of the tectum for each dimension. Because isoelevation contours did not run parallel to the dorsomedial edge, when calculating the magnification factor for elevation, only units with fields centered within $10^{\circ}$ of the midsagittal plane were included $(\mathrm{O}$, Fig. $9 B)$.

In the region of the tectum that represents the frontal $20^{\circ}$ of space (i.e., the directions receiving the greatest representation), the average magnification factors were $150 \mu \mathrm{m} / \mathrm{deg}$ in azimuth and $90 \mu \mathrm{m} / \mathrm{deg}$ in elevation. Along the representation of the midsagittal plane (vertical meridian), which remains within the binocular zone, the magnification factor remained essentially constant (Fig. 9B). However, along the anteroposterior axis of the tectum, the magnification factor for the representation of azimuths decreased substantially about $4 \mathrm{~mm}$ back from the anterior end where azimuths between $10_{\mathrm{c}}^{\circ}$ and $20_{\mathrm{c}}^{\circ}$ were represented (Fig. $9 \mathrm{~A}$ ). Beyond $20_{\mathrm{c}}^{\circ}$, which corresponds to the monocular field, the magnification factor remained relatively constant at $50 \mu \mathrm{m} / \mathrm{deg}$ of azimuth.

Variation in visual receptive field sizes. The receptive fields of hash responses recorded in the superficial layers typically subtended less than $2^{\circ}$ of visual angle, much 

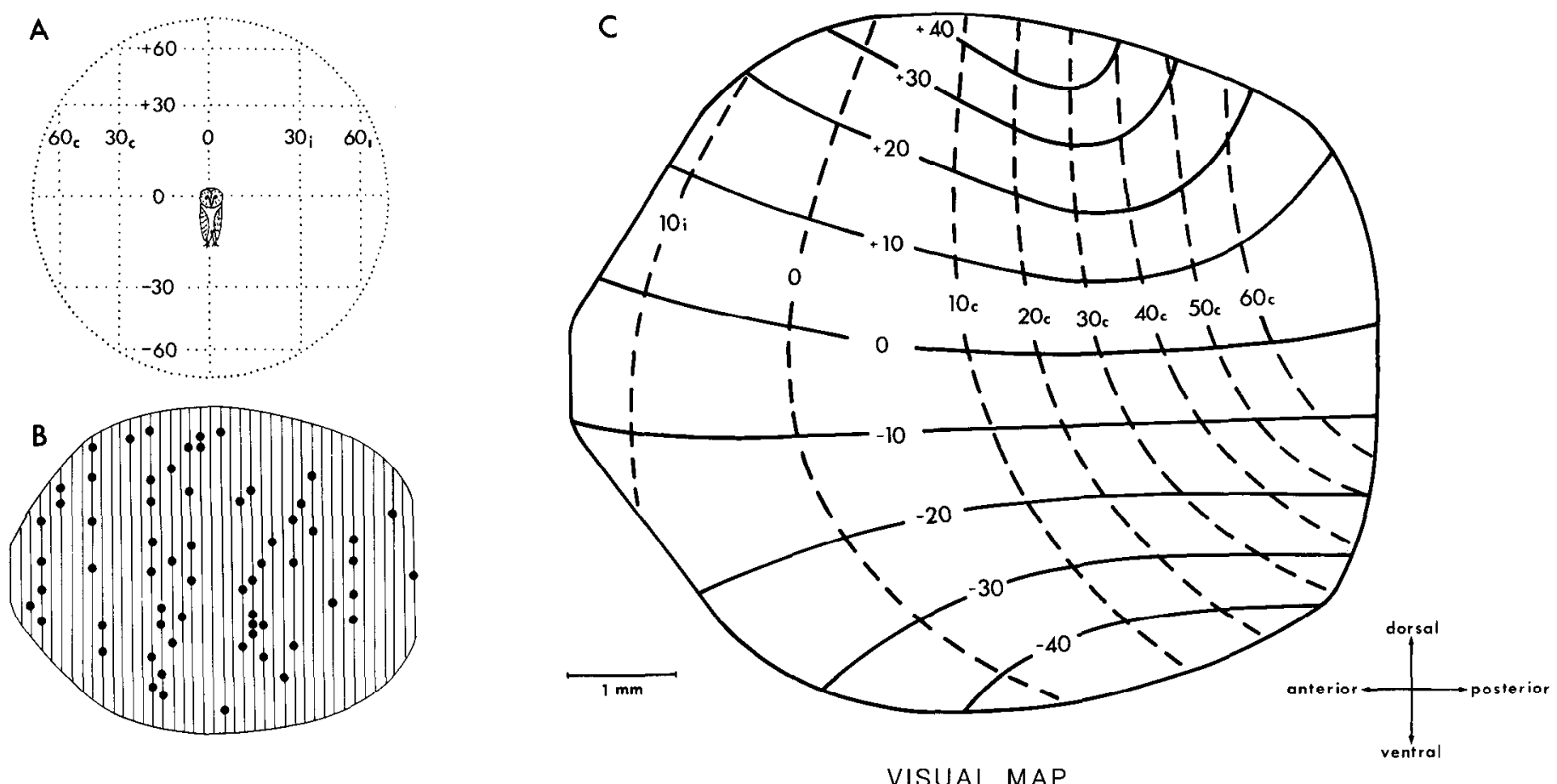

VISUAL MAP

Figure 8. The visual map of space in the optic tectum. $A$, The double pole coordinates of space used in making the map. $B$, The solid circles show the locations of the 63 reconstructed recording sites (from eight birds) used in mapping visual space onto the two-dimensional projection of the tectal surface (see Fig. 3). C, Within the outline of the two-dimensional projection are drawn lines connecting the locations of units with visual field centers at the same azimuth (dashed) or elevation (solid). $c$, Contralateral; $i$, ipsilateral.

A

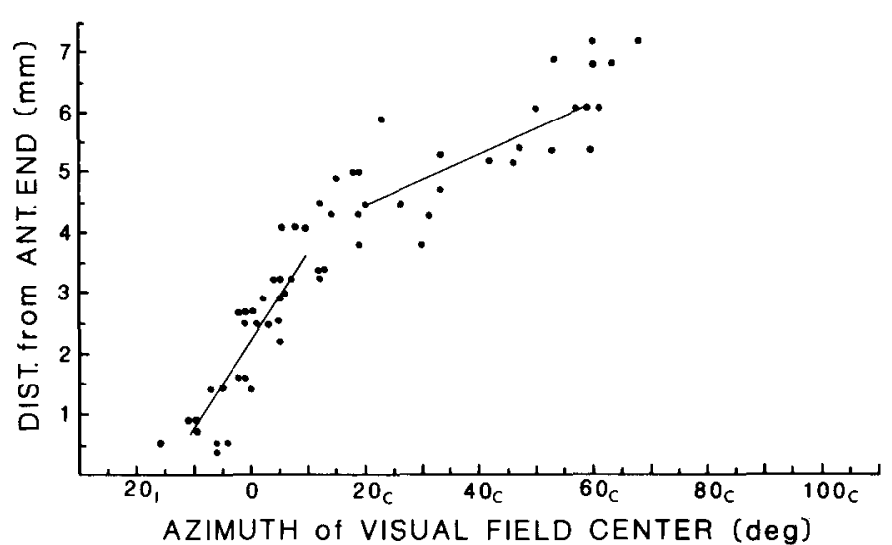

B

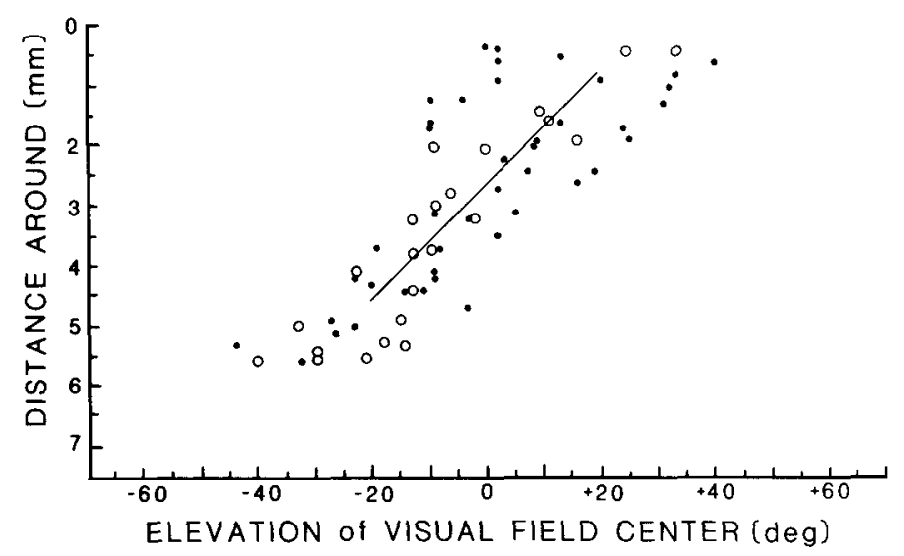

Figure 9. Average magnification factors of the visual map of space. $A$, The distances of units from the anterior end of the tectum are plotted as a function of the azimuth of their visual field centers. The relationship is distinctly nonlinear, with a steep component over the frontal $20^{\circ}$ and a gradual component in the monocular zone (beyond $20_{\mathrm{c}}^{\circ}$ ). The lines are best fits to the points from $10_{\mathrm{i}}^{\circ}$ to $10_{\mathrm{c}}^{\circ}$ and $20_{\mathrm{c}}^{\circ}$ to $60_{\mathrm{c}}^{\circ}$. All elevations are combined. The slopes of these lines represent the average magnification factors in these regions. $B$, The distances of units from the dorsomedial edge of the tectal projection (Fig. 3) are plotted as a function of the elevation of their visual field centers. The average magnification factor was computed from units having fields within $10^{\circ}$ of the midsagittal plane $(O)$. The magnification factor for elevation along the midsagittal plane is relatively constant.

less than the fields of nearby (bursty) units. The receptive field widths of bursty units (superficial layers) averaged $12 \pm 7^{\circ}$, those of regular units (deep layers) averaged 13 $\pm 10^{\circ}$, and those of multiunit recordings (deep layers) were $14 \pm 13^{\circ}$. This constancy of field size among units from the superficial and deep layers does not necessarily contradict the rule established by numerous studies in a variety of other species that receptive fields increase in size with recording depth. Bursty unit recordings were usually multiunit recordings, which artificially increased the apparent sizes of the bursty unit receptive fields. In addition, the acoustic search stimulus undoubtedly biased the sample toward bimodally responsive units, which may represent an unusual and select subset of units, particularly in the superficial layers where auditory responses have not been observed in previous studies. 
TABLE I

Receptive field width versus location

\begin{tabular}{|c|c|c|c|c|c|c|}
\hline & \multicolumn{3}{|c|}{$\begin{array}{l}\text { Visual Receptive Fields: } \\
\text { Azimuth of Field Center }\end{array}$} & \multicolumn{3}{|c|}{$\begin{array}{l}\text { Auditory Receptive Fields: } \\
\text { Azimuth of Best Area Center }\end{array}$} \\
\hline & $<10^{\circ}$ & $10^{\circ}-20^{\circ}$ & $>20^{\circ}$ & $<10^{\circ}$ & $10^{\circ}-20^{\circ}$ & $>20^{\circ}$ \\
\hline \multicolumn{7}{|l|}{$\begin{array}{l}\text { Bursty units } \\
\text { (superficial layers) }\end{array}$} \\
\hline Mean width & 10 & 11 & 16 & 30 & 34 & 48 \\
\hline Standard deviation & 5 & 5 & 8 & 13 & 11 & 15 \\
\hline Sample size & 33 & 13 & 21 & 36 & 14 & 17 \\
\hline \multicolumn{7}{|l|}{$\begin{array}{l}\text { Regular units } \\
\text { (deep layers) }\end{array}$} \\
\hline Mean width & 9 & 12 & 17 & 21 & 31 & 44 \\
\hline Standard deviation & 7 & 7 & 14 & 14 & 10 & 23 \\
\hline Sample size & 33 & 15 & 28 & 30 & 15 & 34 \\
\hline
\end{tabular}

${ }^{a}$ Fields of all elevations are compiled together.

In contrast to the constancy of receptive field size among the different unit types, field sizes of both bursty and regular units increased considerably with the angular distance of their centers away from the midsagittal plane (Table I). Receptive fields located frontally (within $10^{\circ}$ of the midsagittal plane) averaged $10^{\circ}$ across in azimuth for bursty units and $9^{\circ}$ for regular units. Fields centered at azimuths greater than $20^{\circ}$ were almost twice as wide on the average: $16^{\circ}$ for bursty units and $17^{\circ}$ for regular units.

The smallest receptive fields were centered within $10^{\circ}$ of the visual axis. These averaged $10^{\circ}$ in azimuth by $8^{\circ}$ in elevation for bursty units and $9^{\circ}$ by $11^{\circ}$ for regular units.

Auditory receptive fields. Nearly all of the bursty and regular units described so far responded to both auditory and visual stimuli (Figs. 6 and 7). Only sounds originating from within a particular region of space excited these units; sounds located outside of this region, regardless of intensity, failed to elicit responses. This excitatory region of space will be referred to as a unit's auditory receptive field. Auditory receptive fields were mapped by measuring the unit's threshold to noise bursts with the speaker located near the center of the field and then increasing the sound level by $20 \mathrm{~dB}$ and moving the speaker to locations where the unit failed to respond. The coordinates of these locations defined the borders of the receptive field. Inhibitory surrounds, such as those observed routinely for limited field units in the owl's MLD (Knudsen and Konishi, 1978c), were exhibited by some units but definitely not by others.

Auditory receptive fields were sharply limited in both azimuth and elevation. The typical field shape was a vertically oriented ellipse, although some were almost circular and others were irregular or slanted ellipses. No band-shaped fields with unlimited elevational sensitivity (like those found in the MLD) were found in the tectum. The fields ranged in size from $8^{\circ}$ to $110^{\circ}$ in azimuth and from $17^{\circ}$ to $88^{\circ}$ in elevation for regular units and from $17^{\circ}$ to $88^{\circ}$ in azimuth and from $15^{\circ}$ to $94^{\circ}$ in elevation for bursty units. The largest fields were centered more than $30^{\circ}$ from the visual axis and wrapped around behind the owl.
The sizes and locations of auditory receptive fields were largely unaffected by sound intensity: over a $20-\mathrm{dB}$ range (10 to $30 \mathrm{~dB}$ above threshold), field borders rarely changed by more than a few degrees. When field borders were indistinct at low sound levels, increasing the sound's intensity sometimes caused an apparent expansion of the field by up to $10^{\circ}$. However, this "expansion" was probably more a consequence of measurement uncertainty at the lower stimulus level than a true change in the field's size.

In contrast to their dramatic spatial selectivity, the units demonstrated little selectivity for the nature of the sound itself. All types of sounds, including tones, clicks, noises, and various man-made sounds, excited these units. The responses of some units varied erratically to repetitions of the same stimulus, but strong habituation was not encountered (possibly due to the nature of the search stimulus).

A few units responded equally well to sounds from any direction within their fields. However, most units preferred sounds from a more restricted "best area." Usually, the location of a best area was obvious from the vigor of a unit's responses to the search stimulus as the speaker was passed through its field. For a rigorous determination of a best area, spike counts were collected to sounds presented from different locations across the unit's field (Fig. 10). The limits of the best area were defined as the locations at which a half-maximum response was elicited. When a best area limit fell between two sampled points, it was approximated by interpolation. On the average, best areas were half the size of the receptive fields. The center of a best area was rarely at the geometric center of the receptive field: the best area center was usually below the receptive field center for high fields and above the receptive field center for low fields. In general, best area and receptive field centers aligned better in azimuth, but even this correspondence was lost in many fields that were located beyond about $30^{\circ}$ to the contralateral side.

Best area centers remained constant with sound intensity (Fig. 11). Although best areas were often more definite when mapped with midrange sound intensities than with lower or higher ones, their centers varied by less than $5^{\circ}$ over a $20-\mathrm{dB}$ range ( 10 to $30 \mathrm{~dB}$ above threshold). 

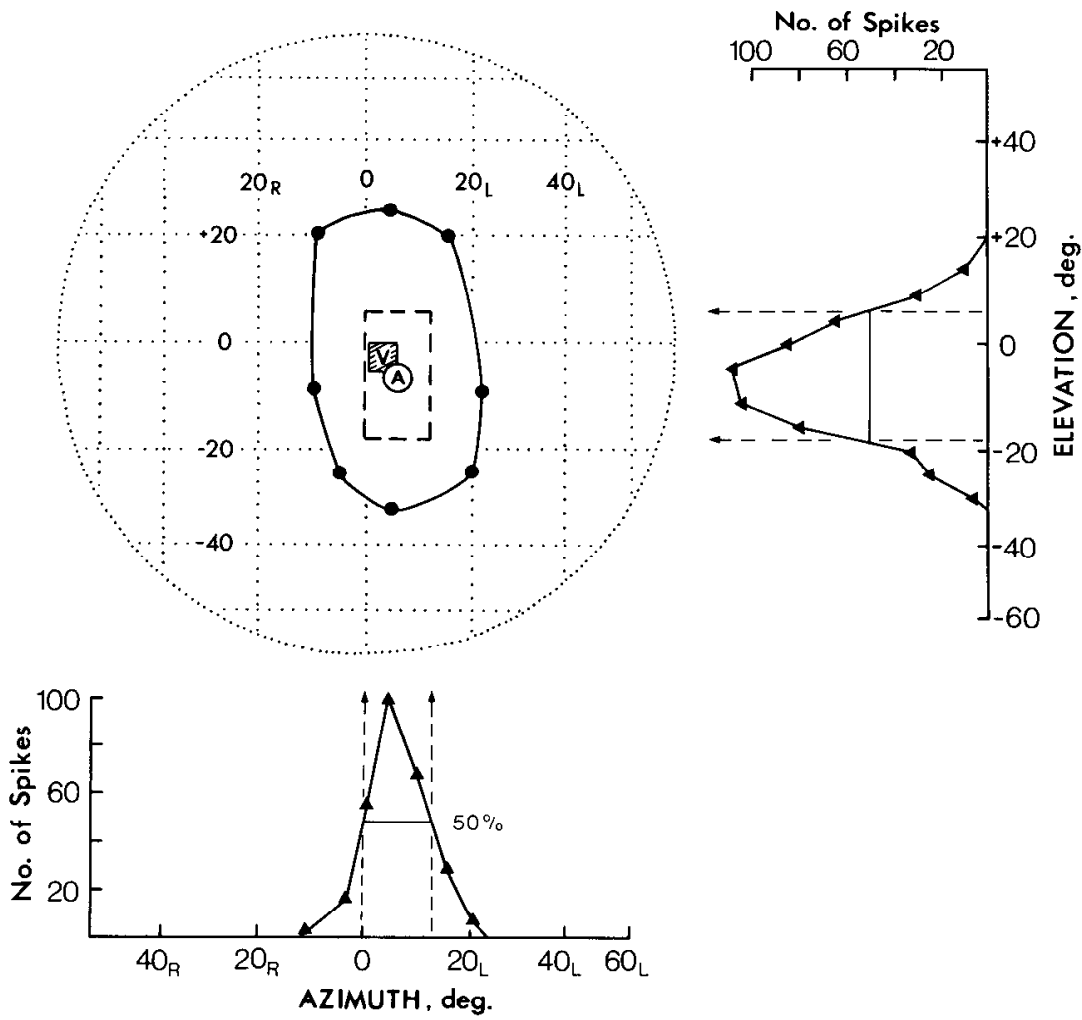

Figure 10. The auditory and visual receptive fields of a bimodal unit. The borders of the auditory field are indicated by solid circles; the visual field is hatched and marked with a $V$ on the dotted globe of double pole space (upper left). The unit's auditory best area is defined as the region of space in which a noise burst was elicited in $50 \%$ of the maximum number of spikes and is marked by the dashed lines on the globe. The center of the best area is indicated by the encircled $A$. The response of the unit (number of spikes) to 10 repetitions of a standard noise burst is plotted as a function of speaker location in azimuth (below) and elevation (right). The noise was $20 \mathrm{~dB}$ above threshold.
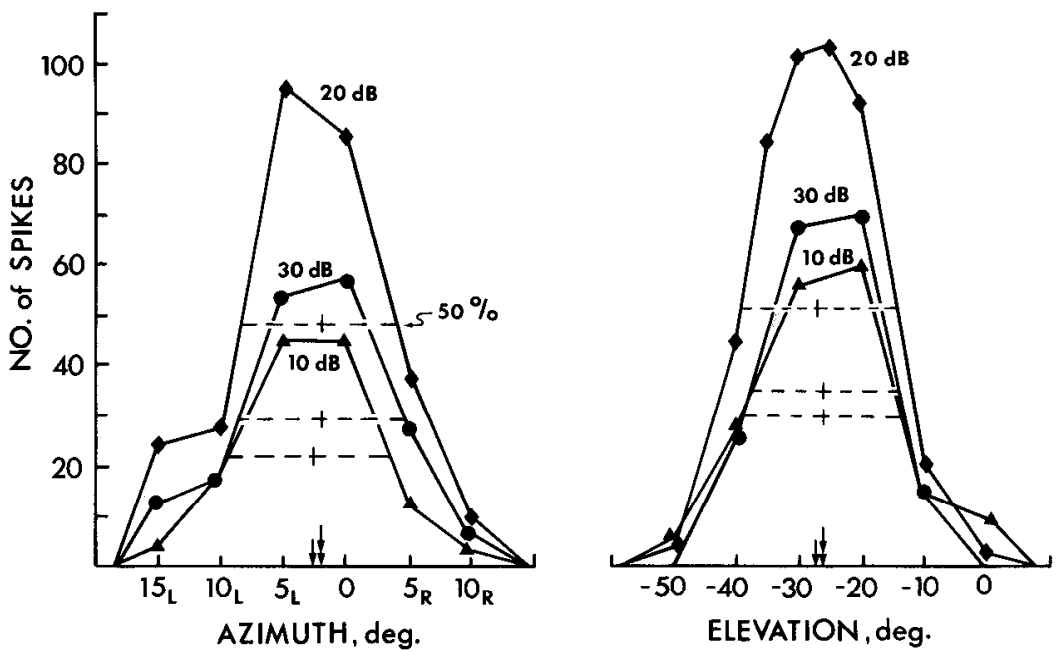

Figure 11. The effect of sound intensity on the spatial response profiles of a bimodal tectal unit. The unit's response (number of spikes) to 10 presentations of the standard noise burst at 10,20, and $30 \mathrm{~dB}$ above threshold are plotted as a function of speaker location in azimuth (left) and elevation (right). The extent of the unit's best area (50\% response) at each intensity is indicated by the dashed lines. The centers of these best areas are marked by the arrows on the abscissa. Sound intensity has virtually no effect on the position of the best area center. Note the change in scale for degrees of azimuth and elevation.

A sound level of $20 \mathrm{~dB}$ above threshold proved to be optimal for a majority of units and subsequently was adopted as the standard level when measuring best areas.

Auditory topography. The locations of receptive fields and best areas systematically correlated with unit posi- tion in the tectum. During dorsoventral penetrations, fields and best areas always began above $0^{\circ}$ elevation and moved downward as the electrode advanced (Figs. 6 and 7). Penetrations through the anterior tectum yielded fields that were ipsilateral to the recording site; penetra- 
tions through the posterior tectum yielded fields that were far to the contralateral side, occasionally even behind the owl's head.

To quantify the topography of the auditory representation of space, single azimuth and elevation values were assigned to each unit based on its best area center (Fig. 11). Units without best areas were assigned the coordinates of their receptive field centers. These values were entered on the flattened map of the tectum, and isoazimuth and isoelevation contours were derived just as they were for the visual map (the same 63 units were used for both maps).
Mapping data obtained from different animals were generally in agreement, particularly in the central portion of the tectum representing $55_{\mathrm{i}}^{\circ}$ to $20_{\mathrm{c}}^{\circ}$ in azimuth and $\pm 20^{\circ}$ in elevation. The greatest discrepancies in the data occurred in the posterior and ventral regions where the representation of space is relatively compressed (Fig. 12). Posteriorly, the azimuths of some best areas deviated by as much as $20^{\circ}$ from the value given on the map; ventrally, elevations deviated by up to $15^{\circ}$ from the illustrated value. In the central portion of the map, the data were very consistent and the contours were accurate to within a few degrees for all units.

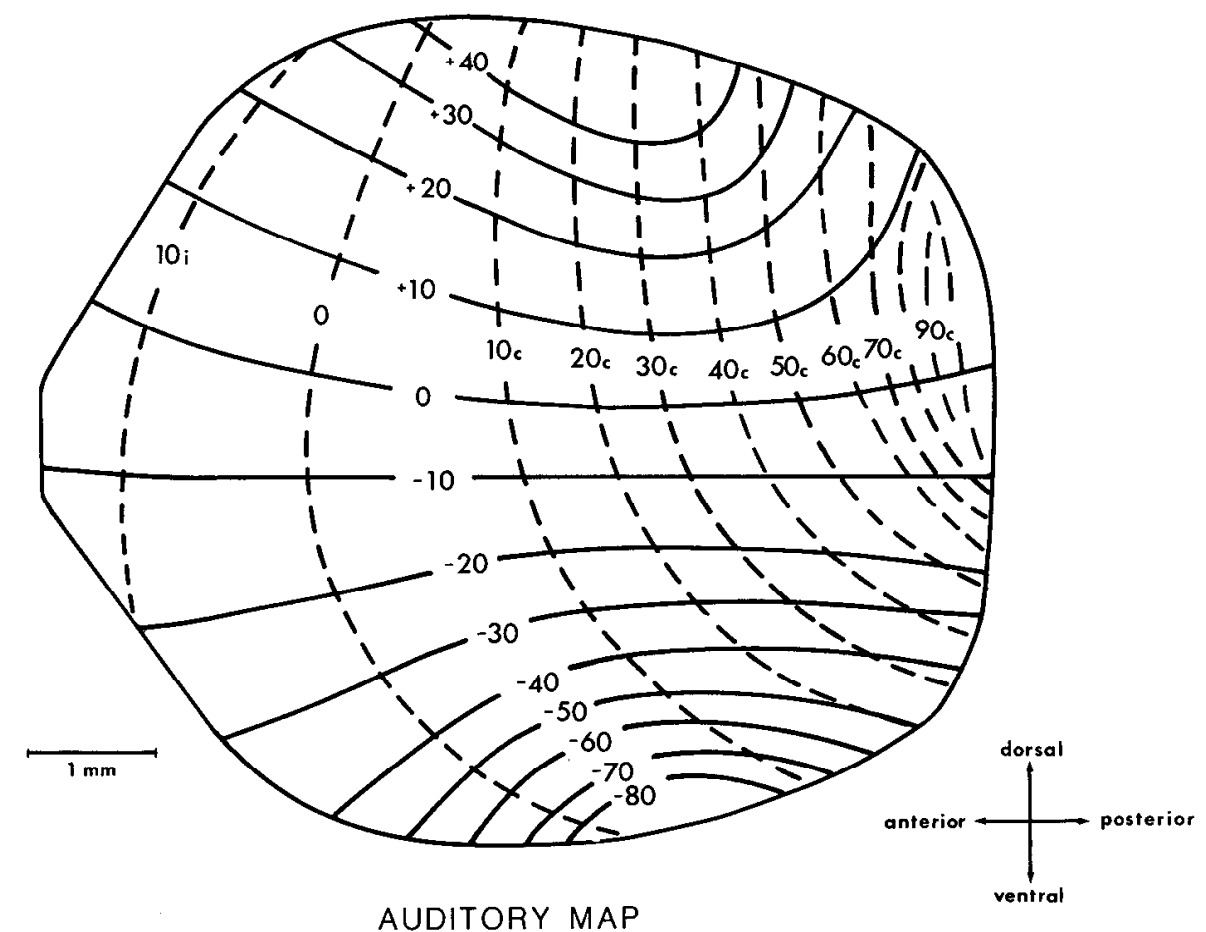

Figure 12. The auditory map of space in the optic tectum. Within the outline of the two-dimensional projection of the tectal surface are drawn lines connecting units with auditory best area centers located at the same azimuth (dashed) or elevation (solid). The map is based on the responses of the same 63 units (from eight birds) used in establishing the visual map (Fig. 8 ). The isoazimuth and isoelevation values are in double pole coordinates.

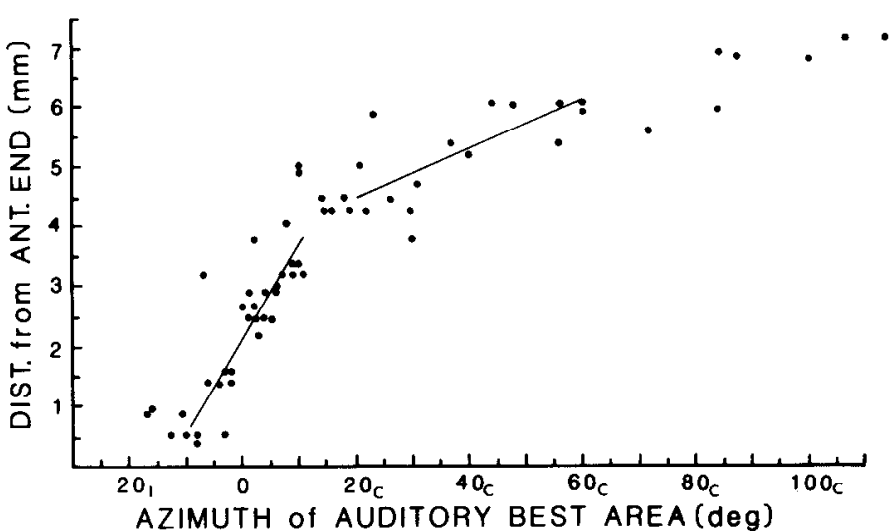

B

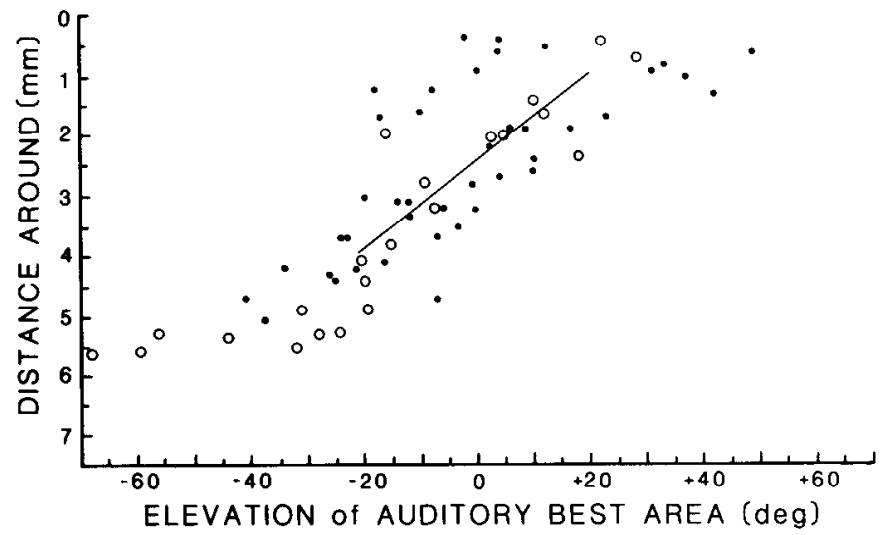

Figure 13. Average magnification factors of the auditory map of space. Compare with Figure 9. A, Distances of units from the anterior end of the tectum are plotted against the azimuth of their auditory best area centers. The slopes of the best fit lines represent the average magnification factors from $10_{\mathrm{i}}^{\circ}$ to $10_{\mathrm{c}}^{\circ}$ and $20_{\mathrm{c}}^{\circ}$ to $60_{\mathrm{c}}^{\circ}$. B, Distances of units along the tectal surface from the dorsomedial edge are plotted against the elevation of their best area centers. The best fit line was based only on units with fields less than $10^{\circ}$ from the midsagittal plane $(O)$. These data are from the same units as used in Figure 9. 
The auditory map of space based on best area centers extended at least from $17_{\mathrm{i}}^{\circ}$ to $115_{\mathrm{c}}^{\circ}$ in azimuth and from $+40^{\circ}$ to $-80^{\circ}$ in elevation. In terms of receptive fields, however, the entire contralateral hemisphere and $30^{\circ}$ into the ipsilateral hemisphere were represented in the tectum. Units responding to directions behind the owl were located at the dorsal, ventral, and posterior edges of the tectum. The fields of these units were large, and though their best areas usually were located in front, their fields continued behind the head. Only at the posterior pole of the tectum were units found with best areas centered behind the head at $\left[100_{c}^{\circ}, 0^{\circ}\right],\left[115_{c}^{\circ},+2^{\circ}\right]$, and $\left[107_{\mathrm{c}}^{\circ},-8^{\circ}\right]$; all were bimodal regular units (Fig. $14 C$ ).

Isoazimuth contours in the dorsal half of the tectum ran approximately dorsoventrally; in the ventral half, they bent posteriorly (Fig. 12). Penetrations made 2 to 3 $\mathrm{mm}$ back from the anterior pole encountered units with fields along the midsagittal plane. The best area of the first unit could be as high as $+40^{\circ}$, depending on the mediolateral placement of the electrode. As the electrode advanced, subsequent best areas descended along about the same azimuth to as low as $-40^{\circ}$. Units with very low best areas $\left(-40^{\circ}\right.$ to $\left.-80^{\circ}\right)$ were located more posteriorly and along the ventromedial margin of the tectum.

Bending of the isoazimuth contours in the ventral half of the tectum was evident from penetrations made more than $3 \mathrm{~mm}$ from the anterior pole (Fig. 7). A penetration made $4.5 \mathrm{~mm}$ back initially encountered bursty units with best areas centered at $\left[25_{\mathrm{c}}^{\circ},+35^{\circ}\right]$. After $170 \mu \mathrm{m}$ of electrode advance, the best area of a regular unit was centered at $\left[23_{\mathrm{c}}^{\circ},+2^{\circ}\right]$. After another $200 \mu \mathrm{m}$, field locations had moved in azimuth to $\left[8_{\mathrm{c}}^{\circ},-40^{\circ}\right]$. More posteriorly, this azimuthal shift in best area became increasingly pronounced to a point where, in the posterior $2 \mathrm{~mm}$ of tectum, best areas moved as much in azimuth as they did in elevation. Because of this bending of isoazimuthal contours, nearly all of the medial edge of the ventral tectum was devoted to the lower portions of frontal space between $5_{\mathrm{i}}^{\circ}$ and $15_{\mathrm{c}}^{\circ}$ (Fig. 12).

Azimuths near the midsagittal plane were represented in a disproportionately large area of the tectum. The zone of binocular overlap occupied $55 \%$ of the auditory map (compared with $60 \%$ in the visual map). The regions of space within $10^{\circ}$ of the visual axis occupied $13 \%$ of the tectum.

When evaluating the auditory (and visual) maps, the distortions caused by the flattening procedure must be kept in mind (see "Materials and Methods"). The most severe distortions occur at the edges of the map near the anterior and posterior ends. The apparent expansions of representation that appear in these portions of the map are mostly artifacts of these distortions.

Magnification factors for the auditory map of space were estimated using the same method as was used for the visual magnification factors. Best area coordinates were plotted as a function of unit position in the tectum, and regression lines were fitted to intervals over which the point scatters appeared linear (Fig. 13). Again for elevation, only data from fields located within $10^{\circ}$ of the midsagittal plane were used ( $O$, Fig. $13 B)$.

As the similarity of the maps suggests, the auditory and visual magnification factors were mostly the same: $150 \mu \mathrm{m} / \mathrm{deg}$ of azimuth within the binocular zone, drop- ping to $50 \mu \mathrm{m} / \mathrm{deg}$ of azimuth in the monocular zone between $20_{\mathrm{c}}^{\circ}$ and $60_{\mathrm{c}}^{\circ}$ (Fig. 13A). The auditory representation of directions beyond $60_{\mathrm{c}}^{\circ}$ were compressed even further, but this portion of the tectum was not sampled sufficiently to estimate a magnification factor accurately. The only difference in the auditory and visual magnification factors was in the representation of elevations along the midsagittal plane: the magnification factor was $70 \mu \mathrm{m} / \mathrm{deg}$ for auditory best areas versus $90 \mu \mathrm{m} / \mathrm{deg}$ for visual receptive fields.

Variation in auditory receptive field sizes. As was true of visual fields, auditory fields did not increase in size with recording depth. The fields of bursty units (superficial layers) averaged $37^{\circ} \pm 13^{\circ}$ in azimuth and $54^{\circ} \pm$ $18^{\circ}$ in elevation, while those of regular units (deep layers) averaged $34^{\circ} \pm 12^{\circ}$ in azimuth and $51^{\circ} \pm 18^{\circ}$ in elevation. The fields did, however, vary in size as a function of their location in space. Auditory fields with best areas centered within $10^{\circ}$ of the visual axis were smaller on the average than fields located anywhere else. In this region, bursty unit fields averaged $24^{\circ} \pm 8^{\circ}$ in azimuth and $40^{\circ} \pm 11^{\circ}$ in elevation, and regular unit fields averaged $19^{\circ} \pm 9^{\circ}$ in azimuth and $44^{\circ} \pm 15^{\circ}$ in elevation. Outside of this frontal region, field sizes tended to increase progressively with angular distance of the best area center from the midsagittal plane (Table I). The average field width of bursty units increased from $30^{\circ}$ to $48^{\circ}$, and that of regular units increased from $21^{\circ}$ to $44^{\circ}$, as best area centers moved in azimuth from within $10^{\circ}$ to more than $20_{\mathrm{c}}^{\circ}$ from the midsagittal plane. Over this same range, the average elevational dimension of the fields remained essentially constant for both types of units.

Alignment of auditory and visual receptive fields. Visual receptive fields were contained totally within the boundaries of the auditory fields for $90 \%$ of the bimodal units. In only 15 out of 144 recordings did any portion of a unit's visual field extend beyond the auditory field, and in only 4 of these were the fields completely non-overlapping. Units with such poorly aligned fields were distributed in nearly all portions of the tectum except in the anterior portion where directions within $10^{\circ}$ of the visual axis were represented.

Even though auditory and visual fields usually overlapped entirely, the alignment was often not perfect. To quantify the degree of misalignment, the center of a unit's visual field was compared with the center of its auditory best area. Auditory best areas were chosen instead of auditory field centers because: (1) best areas were well defined even for units with large or irregular auditory fields for which "centers" were ambiguous and (2) best areas and visual field centers corresponded more closely than did auditory and visual field centers, especially when the auditory field was located peripherally and wrapped around behind the owl (Fig. 14). The resulis from the analysis of field alignment were the same for bursty and regular units and hence have been combined in this presentation.

The auditory and visual fields of a unit were considered to be "aligned" when the centers of the auditory best area and visual field were located within $2^{\circ}$ of each other. Based on this criterion, the fields of $35 \%$ of the units (out of 144) were aligned. More than half of these fields were centered within $10^{\circ}$ of the visual axis, but some fields 
located out as far as $60_{\mathrm{c}}^{\circ}$ in azimuth or $\pm 32^{\circ}$ in elevation were aligned perfectly.

Units with misaligned fields occurred throughout the tectum, but they occurred more frequently in areas away from the representation of the visual axis. This trend is readily seen in Figure 15 where the locations of auditory best area centers are plotted against visual field centers. The proportion of units with misaligned fields increased from $57 \%$ for auditory centers having azimuths of less than $10^{\circ}$ to $69 \%$ for azimuths between $10_{\mathrm{c}}^{\circ}$ and $30_{\mathrm{c}}^{\circ}$ and to $73 \%$ for azimuths greater than $30 \%$. The percentage of units with misaligned fields climbed even faster with increasing or decreasing elevations: $51 \%$ among units with auditory field elevations of less than $10^{\circ}, 71 \%$ for elevations between $10^{\circ}$ and $30^{\circ}$ (up or down), and $90 \%$ for elevations below $-30^{\circ}$.

The degree to which auditory and visual fields were misaligned remained about the same for fields centered within $30^{\circ}$ of the visual axis. The median misalignment within this region was $3^{\circ}$ (range, $19^{\circ}$ ) in azimuth and $5^{\circ}$ (range, $14^{\circ}$ ) in elevation. Units with auditory centers more than $30^{\circ}$ from the midsagittal plane exhibited a median field misalignment of $6^{\circ}$ (range, $23^{\circ}$ ) in azimuth and $3^{\circ}$ (range, $13^{\circ}$ ) in elevation, while fields above or below $30^{\circ}$ in elevation had median misalignments of $2^{\circ}$ (range, $3^{\circ}$ ) in azimuth and $7^{\circ}$ (range, $22^{\circ}$ ) in elevation. Notice that only azimuthal alignment deteriorated at large azimuths, and only elevational alignment deteriorated at large elevations.

There was no overall bias in the direction of misalignment for fields centered above $-10^{\circ}$ in elevation and less than $40_{\mathrm{c}}^{\circ}$ in azimuth (Fig. 15). This indicates that, in this region of the tectum, despite random unit to unit variability in the precision of bimodal field alignment, the auditory and visual maps of space were essentially in register.

A distinct bias in the direction of misalignment appeared among units with auditory centers lower than
A

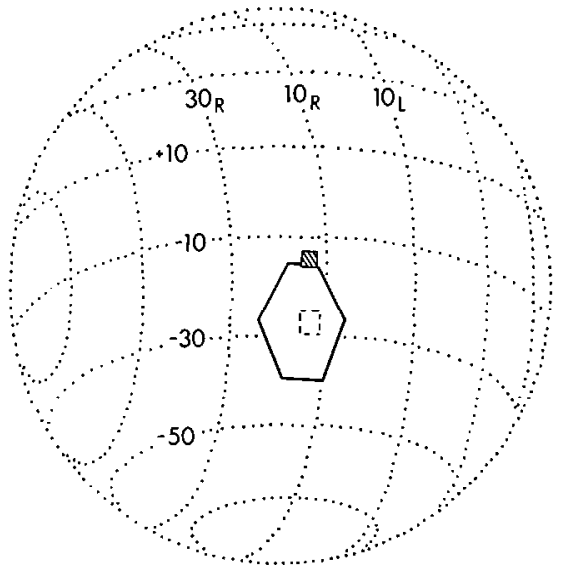

B

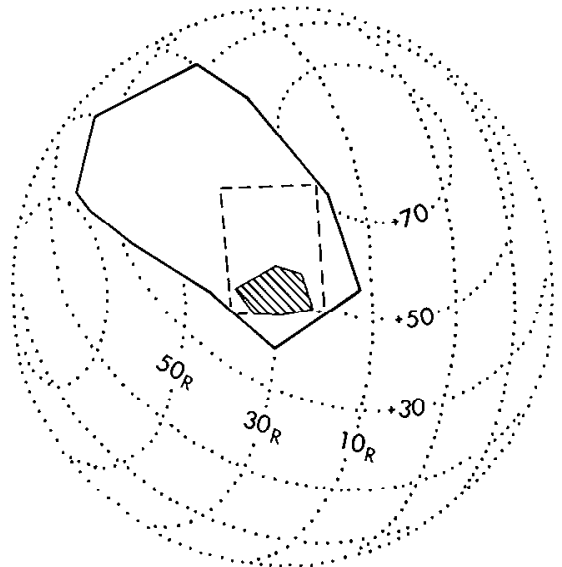

C

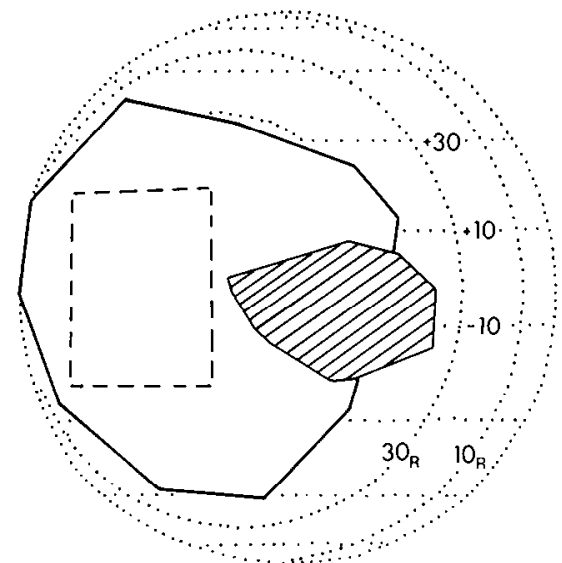

Figure 14. Poorly aligned visual and auditory receptive fields of three bimodal units. The visual fields are hatched; the auditory fields are bounded by bold solid lines; the auditory best areas are represented by dashed lines. The response areas are projected into double pole grids that have been rotated to various degrees: $(A) \mathrm{L} 20_{\mathrm{az}}^{\circ},+20_{\mathrm{e} 1}^{\circ} ;(B) \mathrm{L} 30_{\mathrm{az}}^{\circ},-50_{\mathrm{el}}^{\circ}$; and $(C) \mathrm{L} 70_{\mathrm{az}}^{\circ}, 0_{\mathrm{e}}^{\circ}$. Note that the auditory best area of $C$ is located behind the owl.

A

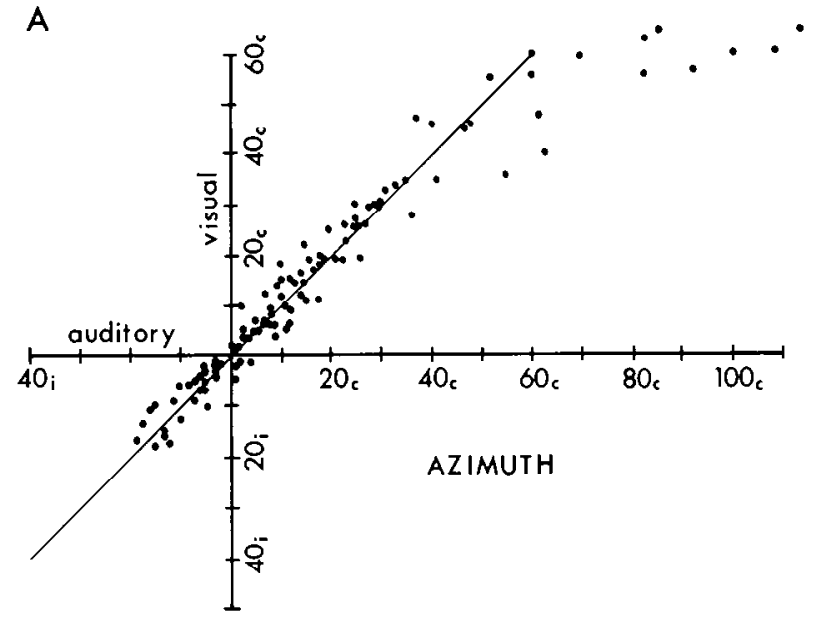

B

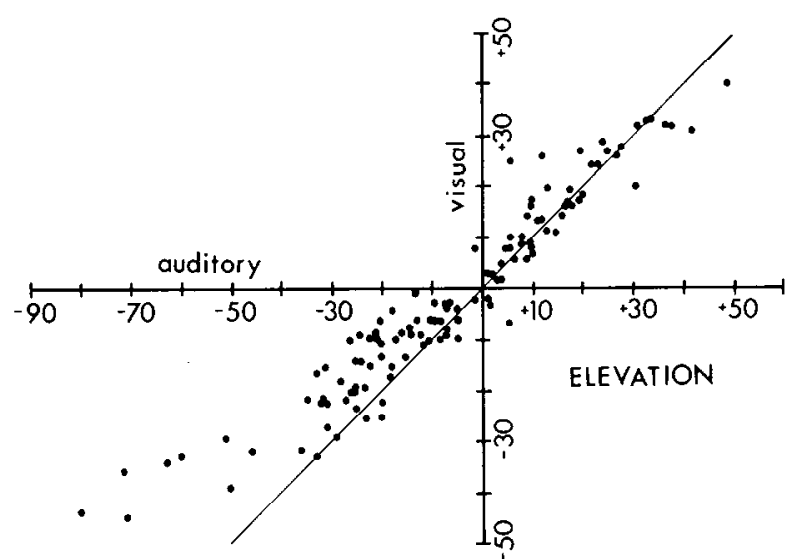

Figure 15. Spatial alignment of the auditory and visual response areas of bimodal units. The visual field center of each unit is plotted as a function of its auditory best area center for azimuth $(A)$ and elevation $(B)$. Points that fall on the $45^{\circ}$ lines represent units with auditory and visual fields that are perfectly aligned in that dimension. The distance of a point away from the line corresponds to the misalignment between a unit's auditory and visual fields. A bias in the distribution of points to one side of the $45^{\circ}$ line indicates a misalignment in the registration of the two sensory maps. 
$-10^{\circ}$ or greater than $40_{\mathrm{c}}^{\circ}$ (Fig. 15). In these regions of the tectum, the auditory map of space continued substantially beyond the owl's visual range (Fig. 5) and therefore represented more of space than did the visual map. Accordingly, $90 \%$ of the units with auditory centers below $-10^{\circ}$ had auditory best areas that were lower than their visual fields, and $85 \%$ of the units with fields greater than $40_{\mathrm{c}}^{\circ}$ had auditory best areas that were further contralateral than their visual fields. This bias in misalignments corresponds to the compression of the auditory map relative to the visual map in these regions.

\section{Discussion}

The response properties of units in the owl's optic tectum challenge a number of generalizations about the properties of superficial and deep layer tectal units that have been derived from numerous studies on a wide range of species. Typically, units recorded in the superficial layers respond only to visual stimuli, have small to intermediate size receptive fields, and do not habituate to repetitive stimulation. In the owl, the first of these generalizations is contradicted: superficial units are strongly bimodal, responding well to both auditory and visual stimuli. Input to the superficial layers from nonvisual modalities is implied by the modulating influence of infrared simulation on visual responses in pit vipers (Newman and Hartline, 1981) and can be induced by enucleating neonatal hamsters (Rhoades, 1980; Rhoades et al., 1981). However, neither of these examples compares with the situation in the owl where strong visual and auditory inputs coexist naturally.

Previous studies have shown that units in the deep layers typically respond to one of a number of different modalities or are polymodal, have large visual receptive fields, and habituate to repetitive stimulation. In the owl, nearly all (90\%) deep layer units are bimodal, have intermediate size receptive fields, and habituate little to repetitive stimulation.

Though some of these discrepancies may be artifacts of sampling techniques and experimental conditions, I suspect that most reflect true species differences that are somehow related to the highly space-specific nature of auditory responses, the constant alignment of the owl's auditory and visual worlds due to the lack of ear and eye movements, and/or the precision with which the owl orients its head to auditory and visual stimuli.

Bursty activity in the superficial layers. Single units were exceedingly difficult to isolate in the superficial layers of the owl's tectum. The likely explanation for this difficulty is that the cells in these layers are unusually small in diameter (5 to $10 \mu \mathrm{m}$ ) and densely packed, almost like granule cells in the cerebellum. However, the bursty pattern of their discharges is less easily explained. Bursty discharges in the superficial layers in monkeys are mentioned by Cynader and Berman (1972) and in cats by Sterling and Wickelgren (1969). However, neither paper emphasizes this property, suggesting that, in these preparations, bursty activity is not pronounced. In the owl's tectum, bursty discharges are ubiquitous and obvious and can be used as a reliable indicator of electrode posilion. The bursty pattern is recorded only in the superficial layers and persists unaltered at all levels of ketamine anesthesia.
A bursty recording normally consists of several units discharging together in an irregular pattern (Fig. 4). Why the impulses of neighboring units should be synchronized is an intriguing question. LaVail and Cowan (1971) note that, in the chick, cells in the superficial layers often occur in clusters. Some cell clustering also can be seen in the owl tectum. Possibly these clustered cells represent a functional unit, all mutually coupled or driven by a common source. The simultaneity of their discharges might be necessary to drive the large cells in the deeper layers. This hypothesis is consistent with the observation that regular units in the deep layers discharge single spikes at approximately the interval of the bursts (Fig. 4).

Receptive field size with depth. 'The visual receptive fields of bursty and regular units are approximately equal in size. As pointed out under "Results," this does not necessarily mean that the progressive increase in field size with unit depth that has been observed in the tecta of all other species does not also occur in the owl. Assuming the fields of nearby bursty units vary slightly in size and location, the multiunit recordings used when mapping these receptive fields artificially enlarged their apparent size. Thus, the fields of single bursty units might be smaller than the fields of regular units.

However, the outstanding aspect of the visual fields in the owl is not the large sizes of fields recorded in the superficial layers but the unusually small sizes of fields recorded in the deep layers. For both bursty and regular units, the average width of receptive fields located frontally remained at about $10^{\circ}$ (Table I). On the other hand, the average width of visual fields in monkeys, cats, rabbits, mice, and pigeons ranges from $5^{\circ}$ to $10^{\circ}$ for superficial units up to $20^{\circ}$ or more for deep layer units (Goldberg and Wurtz, 1972; Gordon, 1973; Graham et al., 1982; Dräger and Hubel, 1975; Hughes and Pearlman, 1974). Perhaps the visual fields in these other species expand to match the large receptive fields of the nonvisual inputs with which they are converging in the deep layers (Gordon, 1973; Dräger and Hubel, 1975; Chalupa and Rhoades, 1977; Harris et al., 1980). In the owl, the visual fields of deep units may remain small, because the extravisual (auditory) fields are small.

Bimodality. Reports on the percentage of units in the deep layers that are polymodal range from $8 \%$ in monkeys (Cynader and Berman, 1972) to $61 \%$ in cats (Gordon, 1973). In other species, the percentage falls somewhere in between. Most units are unimodal, responding to one of several different modalities and are grouped according to their modality sensitivity. Auditory only and auditoryvisual units do not appear to be distributed evenly throughout the tectum but are concentrated in the posterior or lateral portions in cats, mice, hamsters (Gordon, 1973; Dräger and Hubel, 1975; Chalupa and Rhoades, 1977), and possibly monkeys (Goldberg and Wurtz, 1972). Auditory responses are rarely or never seen in the anterior tectum where frontal space is represented.

In owls, $89 \%$ of the units in the deep layers were bimodal (searched for with an auditory stimulus), and these bimodal units occurred throughout the anteroposterior and mediolateral extent of the tectum. This atypical ubiquity of auditory representation may be a consequence of two properties of the owl's auditory and visual 
systems. First, the ears and eyes do not move in the head so that the owl's auditory and visual worlds remain aligned. This would not be the case for vertebrates with movable ears or eyes (most mammals). Secondly, the owl's auditory system is capable of high spatial resolution that can supplant or substantially augment the spatial information provided by the visual system (Konishi, 1973). In contrast the spatial information offered by the auditory systems of less specialized animals may be crude and serve only to indicate the general direction of a stimulus. If converged extensively with visual inputs, this information might blur rather than sharpen the spatial image of the stimulus event.

Topography of space representation. The neural representation of auditory space depends on neuronal specificities for the timing and intensity of sound at the two ears (Moiseff and Konishi, 1981), while the representation of visual space depends on point-to-point projections from the retinal surface. Despite these very different ways in which spatial information is derived, auditory and visual space are basically aligned in the tectum: the maps of space are in the same orientations and absolute positions, and they apportion representation of various regions in space in approximately the same way (Figs. 8 , 9,12 , and 14). This suggests that space per se is the major determinant of functional organization in the tectum. On the other hand, there is a substantial amount of jitter in the alignment of fields of individual units, and actual misalignment in the representations of extreme contralateral and inferior space (Fig. 15). The combination of these observations suggests that spatiotopic organization in the tectum is a compromise between a tendency for the space representations of different modalities to align and for the representation of each modality to fill the entire tectum.

The tendency for space representations to align is evidenced by the equal magnification factors and common termination coordinates of the visual and auditory maps. The expansion of space representation near the visual axis is a common feature of retinotopic projections throughout the visual system and is due in large part to the increased receptor density in the area centralis or fovea. However, there is no comparable, physical explanation for the equivalent expansion of auditory space representation near the visual axis. Studies by Moiseff and Konishi (1981) indicate that the azimuthal boundaries of limited field units are dictated largely by interaural time criteria; and for azimuths out to $60_{\mathrm{c}}^{\circ}$, interaural time varies almost linearly with angle. Yet over this same range of directions, the magnification factor in the auditory map drops from 150 to $50 \mu \mathrm{m} / \mathrm{deg}$, mimicking the change in the visual magnification factor.

In addition, both maps of space stop at $20^{\circ}$. This coordinate corresponds to the visual limit of the contralateral eye (Fig. 5) and demonstrates that, in the owl, just as in all subprimate vertebrates, the entire visual field of the contralateral eye is represented in each tectum. However, why should the auditory map terminate at the same spatial coordinate? There is no discontinuity in localization cues that predicts such a division of auditory space.

These coincidences in the sensory maps imply that either the auditory map follows and conforms to the visual map per se or that both maps are dictated by a third. The third map may be intrinsic to the tectum or established by the motor system (i.e., the motor map of space; Schiller and Koerner, 1971; Schiller and Stryker, 1972; Mays and Sparks, 1980).

The systematic misalignment of auditory and visual space in the posterior and ventral portions of the tectum demonstrates that "space matching" cannot be the only determinant of functional organization. In these portions of the tectum, the auditory map extends beyond the visual map to directions in which the owl cannot see. A simple space-matching hypothesis would predict that bimodal fields should remain aligned out to the limits of vision. If a "third map" were to continue beyond this point, then the visual representation should end and only the auditory representation would persist to the edges of the tectum.

This does not occur. Instead, the alignment of auditory and visual fields becomes progressively worse as the fields are located farther contralateral and down, with auditory fields always located peripheral to visual fields (Fig. 15). This compression of the auditory map relative to the visual map suggests a tendency for each modality to fill the tectum with its representation of space, a tendency that, in the posterior and ventral tectum, gradually prevails over the tendency to space match.

Space coding in the tectum. Auditory receptive fields are much larger than visual fields; that is, a single unit responds to a larger region of auditory than visual space. Obviously then, the activity of a unit does not by itself code for the location of a stimulus in space.

A more likely space code is one based on relative neural activity within a population of units. The larger size of the auditory fields means that a correspondingly larger population of units will be activated by a sound than by a light stimulus. However, because the units exhibit strong best areas (Figs. 10 and 11), there will exist within the population a steep gradient of excitation, the peak of which will be limited to a circumscribed portion of the tectum. The alignment of the maps indicates that the site of peak activity will correspond to the same location in space for both modalities. Moreover, the amount of tectum maximally activated may be the same for sound and light stimuli; with best area defined as the region to which a unit responds with a greater than halfmaximal response, best areas were only slightly larger than visual receptive fields.

This hypothesis implies that the most significant neural parameter for space coding in the tectum is the change in unit response rates with stimulus location. Although the appropriate measurements (i.e., spatial response profiles and best areas) were made using acoustic stimuli, they were not made using light stimuli. Visual best areas were observed frequently, however, and have been documented for tectal units in other species (Wickelgren, 1971; Goldberg and Wurtz, 1972). Perhaps, if visual best areas were quantified and substituted for visual field centers, the agreement between the auditory and visual space maps would improve still further.

Misaligned receptive fields. A perplexing question is: how does the brain interpret the activity of bimodal units with grossly misaligned or non-overlapping receptive fields? Such units occur throughout the tectum (except near the representation of the visual axis) but are most 
common in the posterior and ventral portions where the sensory maps are out of register. Does the brain interpret neural activity in these portions as signifying that a stimulus is located at the peripheral location indicated by the auditory map or at the more frontal location indicated by the visual map; does the brain identify the stimulus modality by some aspect of the pattern of activation and evaluate the spatial information in a modality-specific way? Certainly the source modality would be readily distinguished by the activity of auditory only and visual only units as well as by differences in the spread of activation through the tectum, but why not keep auditory and visual space separate in parallel channels? What purpose does it serve to converge spatially discordant information from different modalities onto single neurons? The answer to these questions may lie in the nature of the connections and interactions of the tectal neurons with motor control neurons in the brainstem and spinal cord.

\section{References}

Allon, N., and Z. Wollberg (1978) Responses of cells in the superior colliculus of the squirrel monkey to auditory stimuli. Brain Res. 159: 321-330.

Bastian, J. (1981) Visual and electrosensory responses in the optic tectum of a weakly electric fish. Soc. Neurosci. Abstr. 7: 845 .

Chalupa, L. M., and R. W. Rhoades (1977) Responses of visual, somatosensory, and auditory neurons in the golden hamster's superior colliculus. J. Physiol. (Lond.) 270: 595-626.

Cooper, M. L., and J. D. Pettigrew (1979) A neurophysiological determination of the vertical horopter in the cat and owl. J. Comp. Neurol. 184: 1-23.

Cowan, W. M., L. Adamson, and T. P. S. Powell (1961) An experimental study of the avian visual system. J. Anat. 95: 545-562.

Cynader, M., and N. Berman (1972) Receptive-field organization of monkey superior colliculus. J. Neurophysiol. 35: 187201.

Dräger, U. C., and D. H. Hubel (1975) Responses to visual stimulation and relationship between visual, auditory and somatosensory inputs in mouse superior colliculus. J. Neurophysiol. 38: 690-713.

Gaither, N. S., and B. E. Stein (1979) Reptiles and mammals use similar sensory organizations in the midbrain. Science 205: 595-597.

Goldberg, M. E., and R. H. Wurtz (1972) Activity of superior colliculus in behaving monkey. I. Visual receptive fields of single neurons. J. Neurophysiol. 35: 542-559.

Gordon, B. (1973) Receptive fields in deep layers of cat superior colliculus. J. Neurophysiol. 36: 157-178.

Graham, J., N. Berman, and E. H. Murphy (1982) Effects of visual cortical lesions on receptive-field properties of single units in superior colliculus of the rabbit. J. Neurophysiol. 47: 272-286.

Harris, L. R., C. Blakemore, and M. Donaghy (1980) Integration of visual and auditory space in the mammalian superior colliculus. Nature 288: 56-59.

Hartline, P. H., L. Kass, and M. S. Loop (1978) Merging of modalities in the optic tectum: Infrared and visual integration in rattlesnakes. Science 199: 1225-1229.

Horn, G., and R. M. Hill (1966) Responsiveness to sensory stimulation of units in the superior colliculus and subjacent tectotegmental regions of the rabbit. Exp. Neurol. 14: 199223.

Hughes, C. P., and A. L. Pearlman (1974) Single unit receptive fields and the cellular layers of the pigeon optic tectum. Brain Res. 80: 365-377.

Knudsen, E. I., and M. Konishi (1978a) Space and frequency are represented separately in the auditory midbrain of the owl. J. Neurophysiol. 41: 870-884.

Knudsen, F. I., and M. Konishi (1978b) A neural map of auditory space in the owl. Science 200: 795-797.

Knudsen, E. I., and M. Konishi (1978c) Center-surround organization of auditory receptive fields in the owl. Science 202: 778-780.

Knudsen, E. I., M. Konishi, and J. D. Pettigrew (1977) Receptive fields of the auditory neurons in the owl. Science 198: $1278-1280$

Knudsen, E. I., G. G. Blasdel, and M. Konishi (1979) Sound localization by the barn owl measured with the search coil technique. J. Comp. Physiol. 133: 1-11.

Konishi, M. (1973) How the owl tracks its prey. Am. Sci. 61: 414-424.

Lane, R. H., J. M. Allman, J. H. Kaas, and F. M. Miezin (1973) The visuotopic organization of the superior colliculus of the owl monkey (Aotus trivirgatus) and the bush baby (Galago senegalensis). Brain Res. 60: 335-349.

LaVail, J. H., and W. M. Cowan (1971) The development of the chick optic tectum. I. Normal morphology and cytoarchitectonic development. Brain Res. 28: 391-419.

Mast, T. E., and D. Y. Chung (1973). Binaural interaction in the superior colliculus of the chinchilla. Brain Res. 62: 227230.

Mays, L. E., and D. L. Sparks (1980) Dissociation of visual and saccade-related responses in superior colliculus neurons. J. Neurophysiol. 43: 207-232.

Moiseff, A., and M. Konishi (1981) Neuronal and behavioral sensitivity to binaural time differences in the owl. J. Neurosci. 1: 40-48.

Newman, E. A., and P. H. Hartline (1981) Integration of visual and infrared information in bimodal neurons of the rattlesnake optic tectum. Science 213: 789-791.

Payne, R. S. (1971) Acoustic location of prey by barn owls (Tyto alba). J. Exp. Biol. 54: 535-573.

Pettigrew, J. D. (1979) Binocular visual processing in the owl's telecephalon. Proc. R. Soc. Lond. (Biol.) 204: 435-454.

Pettigrew, J. D., and M. Konishi (1976) Neurons selective for orientation and binocular disparity in the visual wulst of the barn owl (Tyto alba). Science 193: 675-678.

Ramon y Cajal, S. (1972) Histologie du Système Nerveaux de l'Homme et des Vertébrés, Ed. 2, Vol. II, pp. 196-212, Instituto Ramon y Cajal, Madrid.

Rhoades, R. W. (1980) Effects of enucleation on the functional organization of the superior colliculus in the golden hamster. J. Physiol. (Lond.) 301: 383-399.

Rhoades, R. W., D. D. Della Croce, and I. Meadows (1981) Reorganization of somatosensory imput to superior colliculus in neonatally enucleated hamsters: Anatomical and electrophysiological experiments. J. Neurophysiol. 46: 855-877.

Schiller, P. H., and F. Koerner (1971) Discharge characteristics of single units in superior colliculus of the alert rhesus monkey. J. Neurophysiol. 34: 920-936.

Schiller, P. H., and M. Stryker (1972) Single-unit recording and stimulation in superior colliculus of the alert rhesus monkey. J. Neurophysiol. 35: 915-924.

Stein, B. E., B. Magalhães-Castro, and L. Kruger (1976) Relationship between visual and tactile representations in cat superior colliculus. J. Neurophysiol. 39: 401-419.

Sterling, P., and B. G. Wickelgren (1969) Visual receptive fields in the superior colliculus of the cat. J. Neurophysiol. 32: 115.

Wickelgren, B. G. (1971) Superior colliculus: Some receptive field properties of bimodally responsive cells. Science 173: 69-72. 